CERN-TH/98-85

FERMILAB-PUB-98/093-T

$\mathrm{IC} / 98 / 25$

hep-ph/9804253

\title{
Extracting CKM Phases and $\boldsymbol{B}_{s}-\overline{\boldsymbol{B}}_{s}$ Mixing Parameters from Angular Distributions of Non-Leptonic $B$ Decays
}

\author{
Amol S. Dighe \\ The Abdus Salam International Centre for Theoretical Physics \\ 34100 Trieste, Italy \\ Isard Dunietz \\ Theoretical Physics Division, Fermi National Accelerator Laboratory \\ Batavia, IL 60510, USA \\ Robert Fleischer \\ Theory Division, CERN, CH-1211 Geneva 23, Switzerland
}

\begin{abstract}
Suggestions for efficiently determining the lifetimes and mass difference of the light and heavy $B_{s}$ mesons $\left(B_{s}^{L}, B_{s}^{H}\right)$ from $B_{s} \rightarrow J / \psi \phi, D_{s}^{*+} D_{s}^{*-}$ decays are given. Using appropriate weighting functions for the angular distributions of the decay products (moment analysis), one can extract $\left(\Gamma_{H}, \Gamma_{L}, \Delta m\right)_{B_{s}}$. Such a moment analysis allows the determination of the relative magnitudes and phases of the $\mathrm{CP}$-odd and CP-even decay amplitudes. Efficient determinations of CP-violating effects occuring in $B_{s} \rightarrow J / \psi \phi, D_{s}^{*+} D_{s}^{*-}$ are discussed in the light of a possible width difference $(\Delta \Gamma)_{B_{s}}$, and the utility of this method for $B \rightarrow J / \psi K^{*}, D_{s}^{*+} \bar{D}^{*}$ decays is noted. Since our approach is very general, it can in principle be applied to all kinds of angular distributions and allows the determination of all relevant observables, including fundamental CKM (Cabibbo-Kobayashi-Maskawa) parameters, as well as tests of various aspects of the factorization hypothesis. Explicit angular distributions and weighting functions are given, and the general method that can be used for any angular distribution is indicated.
\end{abstract}

CERN-TH/98-85

April 1998 



\section{Introduction}

Strategies for obtaining experimental insights into CP violation and non-factorizable contributions to weak decays are of particular interest for present particle physics. The observables of angular distributions can be obtained in an efficient way by using an angular moment analysis [1]-[3]. In this approach, the observed experimental data are weighted by judiciously chosen functions, which project out any desired observable. This strategy is an alternative to the usual likelihood fit method [1]. It is demonstrated that the moment analysis extracts all observables of measured angular distributions, such as the ones occurring in weak decays of pseudoscalars $\left[P \rightarrow V \ell \nu, X_{J} \ell \nu, V V\right.$, etc.]. This method is of general validity. In our present paper, we apply the formalism to angular distributions [5, 6] of $B_{s}$ and $B$ meson decays into two vector-meson final states that are caused by $\bar{b} \rightarrow \bar{s} c \bar{c}$ quark-level transitions. By making use of the general formalism outlined in this paper, it is straightforward to derive weighting functions for other exclusive mesonic or baryonic transitions, governed for instance by $b \rightarrow c \bar{u} d, c \ell \bar{\nu}, u \ell \bar{\nu}, c \rightarrow s \bar{d} u, s \ell^{+} \nu, d \ell^{+} \nu$.

The mixing between neutral $B_{s}$ mesons is expected to give rise to CP-even $\left(B_{s}^{L}\right)$ and CP-odd $\left(B_{s}^{H}\right)$ mass eigenstates, which may have a perceptible width difference $\Delta \Gamma \equiv$ $\Gamma_{H}-\Gamma_{L}$ [7]. Using appropriate weighting functions for the angular distributions of the decay products in the transitions $B_{s} \rightarrow J / \psi \phi$ and/or $B_{s} \rightarrow D_{s}^{*+} D_{s}^{*-}$, one can extract $\left(\Gamma_{H}, \Gamma_{L}, \Delta m\right)_{B_{s}}$.

A characteristic feature of the angular distributions considered in this paper is the fact that they contain terms describing interference effects between CP-even and CP-odd final-state configurations. Because of the lifetime difference, these contributions give rise to a term in the time evolution of the untagged rate, which is proportional to [8]:

$$
\left(e^{-\Gamma_{H} t}-e^{-\Gamma_{L} t}\right) \sin \phi_{\mathrm{CKM}},
$$

where $\phi_{\mathrm{CKM}}$ is a weak phase that is introduced through the CKM matrix [9]. In the $B_{s}$ decays considered in the present paper, $\phi_{\mathrm{CKM}}$ is related to the Wolfenstein parameter $\eta$ [10]. It is a remarkable feature that time-evolved untagged data samples of angular distributions of $B_{s}$ decays may exhibit $\mathrm{CP}$-violating effects, if $\Delta \Gamma$ is sizeable [8, 11]. This feature may be important, because it provides an alternative to previous investigations, which have shown how to extract $\sin \phi_{\text {CKM }}$ from tagged, time-dependent analyses [12, 13]. This extraction, however, may not be feasible in the near future because it requires tagging and superb vertex detectors, which must resolve the rapid $\Delta m t$ oscillations. In contrast, any dependence on $\Delta m t$ cancels in untagged data samples, which therefore allow feasibility studies with current vertex technology [14].

Concerning tests of the factorization hypothesis [15]-20], we divide the $\bar{b} \rightarrow \bar{s} c \bar{c}$ modes into the following two categories:

- colour-suppressed decays: $B_{q} \rightarrow J / \psi V$ with $(q, V) \in\left\{(s, \phi) ;\left(d, K^{* 0}\right) ;\left(u, K^{*+}\right)\right\}$ [21, 22].

- colour-allowed decays: $B_{q} \rightarrow D_{s}^{*+} \bar{D}_{q}^{*}$ with $q \in\{s, d, u\}$ [23, 24]. 
Whereas the validity of the factorization assumption is very doubtful in the coloursuppressed case, it should work much better for the colour-allowed channels because of colour transparency [18]. The latter have furthermore rather tight restrictions from the Heavy Quark Effective Theory (HQET) [25] for the form factors describing the "factorized" hadronic matrix elements of the relevant four-quark operators [23, 24, 26].

Our paper is organized as follows: in Section $\mathbb{1}$ we calculate the transition matrix elements and observables of the angular distributions by using an appropriate low-energy effective Hamiltonian. There we also give estimates for these observables, allowing a comparison with experimental data. The efficient experimental determination of these observables is the subject of Section [II], where we shall discuss the angular moment analysis. Sections [V and $\mathrm{V}$ are devoted to the angular correlations in the colour-suppressed decays $B_{s} \rightarrow J / \psi \phi, B \rightarrow J / \psi K^{*}$ and the colour-allowed decays $B_{s} \rightarrow D_{s}^{*+} D_{s}^{*-}, B \rightarrow D_{s}^{*+} \bar{D}^{*}$, respectively. There we give the time evolutions of the angular distributions, appropriate weighting functions, and discuss CP-violating effects. Finally in Section VI the main results are summarized.

\section{Transition matrix elements and observables}

Before we present an efficient method for extracting the observables of the angular distributions from experimental data - the angular moment analysis - let us discuss in this section how these observables are calculated and what orders of magnitude we expect for them.

\section{II-A General aspects}

In order to calculate the decay amplitudes of the $\bar{b} \rightarrow \bar{s} c \bar{c}$ transitions considered in this paper, we use an appropriate low-energy effective Hamiltonian, which has the following structure:

$$
\mathcal{H}_{\mathrm{eff}}=\frac{G_{\mathrm{F}}}{\sqrt{2}}\left[\sum_{j=u, c} \lambda_{j}^{(s)}\left\{Q_{1}^{j} C_{1}(\mu)+Q_{2}^{j} C_{2}(\mu)+\sum_{k=3}^{10} Q_{k} C_{k}(\mu)\right\}\right] .
$$

Here the quantities $\lambda_{j}^{(s)} \equiv V_{j s} V_{j b}^{*}$ denote CKM factors,

$$
\begin{aligned}
Q_{1}^{c} & =\left(\bar{c}_{\alpha} s_{\beta}\right)_{\mathrm{V}-\mathrm{A}}\left(\bar{b}_{\beta} c_{\alpha}\right)_{\mathrm{V}-\mathrm{A},} & Q_{2}^{c} & =\left(\bar{c}_{\alpha} s_{\alpha}\right)_{\mathrm{V}-\mathrm{A}}\left(\bar{b}_{\beta} c_{\beta}\right)_{\mathrm{V}-\mathrm{A}} \\
Q_{1}^{u} & =\left(\bar{u}_{\alpha} s_{\beta}\right)_{\mathrm{V}-\mathrm{A}}\left(\bar{b}_{\beta} u_{\alpha}\right)_{\mathrm{V}-\mathrm{A}}, & Q_{2}^{u} & =\left(\bar{u}_{\alpha} s_{\alpha}\right)_{\mathrm{V}-\mathrm{A}}\left(\bar{b}_{\beta} u_{\beta}\right)_{\mathrm{V}-\mathrm{A}}
\end{aligned}
$$

are "current-current" operators,

$$
\begin{array}{lll}
Q_{3}=\left(\bar{b}_{\alpha} s_{\alpha}\right)_{\mathrm{V}-\mathrm{A}} \sum_{q=u, d, s, c, b}\left(\bar{q}_{\beta} q_{\beta}\right)_{\mathrm{V}-\mathrm{A}}, & Q_{4}=\left(\bar{b}_{\alpha} s_{\beta}\right)_{\mathrm{V}-\mathrm{A}} \sum_{q=u, d, s, c, b}\left(\bar{q}_{\beta} q_{\alpha}\right)_{\mathrm{V}-\mathrm{A}} \\
Q_{5}=\left(\bar{b}_{\alpha} s_{\alpha}\right)_{\mathrm{V}-\mathrm{A}} \sum_{q=u, d, s, c, b}\left(\bar{q}_{\beta} q_{\beta}\right)_{\mathrm{V}+\mathrm{A}}, & Q_{6}=\left(\bar{b}_{\alpha} s_{\beta}\right)_{\mathrm{V}-\mathrm{A}} \sum_{q=u, d, s, c, b}\left(\bar{q}_{\beta} q_{\alpha}\right)_{\mathrm{V}+\mathrm{A}}
\end{array}
$$


describe QCD penguins, while the operators

$$
\begin{array}{rlrl}
Q_{7} & =\frac{3}{2}\left(\bar{b}_{\alpha} s_{\alpha}\right)_{\mathrm{V}-\mathrm{A}} \sum_{q=u, d, s, c, b} e_{q}\left(\bar{q}_{\beta} q_{\beta}\right)_{\mathrm{V}+\mathrm{A}}, & Q_{8} & =\frac{3}{2}\left(\bar{b}_{\alpha} s_{\beta}\right)_{\mathrm{V}-\mathrm{A}} \sum_{q=u, d, s, c, b} e_{q}\left(\bar{q}_{\beta} q_{\alpha}\right)_{\mathrm{V}+\mathrm{A}} \\
Q_{9} & =\frac{3}{2}\left(\bar{b}_{\alpha} s_{\alpha}\right)_{\mathrm{V}-\mathrm{A}} \sum_{q=u, d, s, c, b} e_{q}\left(\bar{q}_{\beta} q_{\beta}\right)_{\mathrm{V}-\mathrm{A}}, & Q_{10}=\frac{3}{2}\left(\bar{b}_{\alpha} s_{\beta}\right)_{\mathrm{V}-\mathrm{A}} \sum_{q=u, d, s, c, b} e_{q}\left(\bar{q}_{\beta} q_{\alpha}\right)_{\mathrm{V}-\mathrm{A}}
\end{array}
$$

are "electroweak" penguin operators. Here $\mathrm{V} \pm \mathrm{A}$ corresponds to $\gamma_{\mu}\left(\hat{1} \pm \gamma_{5}\right)$ quark currents, Greek indices are associated with the $S U(3)_{\mathrm{C}}$ quark-colour, and the quantities $e_{q}$ arising in the expressions for the electroweak penguin operators label the electrical quark charges. Nowadays, the Wilson coefficient functions $C_{k}(\mu)$ of the low-energy effective Hamiltonian $\mathcal{H}_{\text {eff }}$, where $\mu=\mathcal{O}\left(m_{b}\right)$ denotes the usual renormalization scale, are known beyond the leading logarithmic approximation [27].

Since $\lambda_{u}^{(s)}$ is suppressed with respect to $\lambda_{c}^{(s)}$ by a CKM factor $\lambda^{2} R_{b}$, where $\lambda=0.22$ is the Wolfenstein parameter [10] and

$$
R_{b} \equiv \frac{1}{\lambda} \frac{\left|V_{u b}\right|}{\left|V_{c b}\right|}
$$

is constrained by present experimental data to lie within the range $R_{b}=0.36 \pm 0.08$ 28, 29, and since furthermore the current-current operators $Q_{1}^{u}, Q_{2}^{u}$ may contribute only through penguin-like matrix elements to $\bar{b} \rightarrow \bar{s} c \bar{c}$ modes, the corresponding transition amplitudes are dominated to an excellent approximation by the contribution proportional to $\lambda_{c}^{(s)}$ (for a detailed discussion, see [30]). In the penguin operators, we neglect the parts of flavour structure different from $(\bar{c} c)(\bar{b} s)$. Then the number of relevant operators reduces from ten to four and the structure of the decay amplitude simplifies considerably.

In order to implement the factorization hypothesis by factorizing the hadronic matrix elements of the four-quark operators $Q_{k}$ into hadronic matrix elements of quark currents, we have to perform suitable Fierz transformations of the operator basis specified in (3)(5). Beyond the leading logarithmic approximation one has to be very careful in performing such Fierz transformations, as the Wilson coefficients depend both on the form of the chosen operator basis and on the applied renormalization scheme [27]. Since we do not use any specific Wilson coefficients to obtain numerical estimates in this paper, we may perform such Fierz transformations and will use a tilde $\left(^{\sim}\right)$ to indicate Fierz-transformed operators. For a discussion of the renormalization-scheme dependences arising beyond the leading logarithmic approximation and their consistent cancellation in the physical transition amplitudes through certain one-loop matrix elements at $\mu=\mathcal{O}\left(m_{b}\right)$, the reader is referred to Ref. [31.

Let us, in the following two subsections, investigate the structure of the hadronic matrix elements of the low-energy effective Hamiltonian [Eq. (2)] for the exclusive coloursuppressed and colour-allowed decays $B_{s} \rightarrow J / \psi \phi, B \rightarrow J / \psi K^{*}$ and $B_{s} \rightarrow D_{s}^{*+} D_{s}^{*-}$, $B \rightarrow D_{s}^{*+} \bar{D}^{*}$, respectively. 


\section{II-B Colour-suppressed decays}

If we perform a Fierz transformation of the current-current operators specified in (3), the decay amplitude for $B_{q} \rightarrow J / \psi V\left((q, V) \in\left\{(s, \phi) ;\left(d, K^{* 0}\right) ;\left(u, K^{*+}\right)\right\}\right)$ can be written in the following form:

$$
\begin{aligned}
& \left\langle J / \psi(\lambda) V(\lambda)\left|\mathcal{H}_{\mathrm{eff}}\right| B_{q}\right\rangle=\frac{G_{\mathrm{F}}}{\sqrt{2}} V_{c s} V_{c b}^{*} \\
& \quad \times\left[\mathcal{C}_{1}^{\mathrm{eff}}(\mu)\left\langle J / \psi(\lambda) V(\lambda)\left|\tilde{Q}_{1}^{c}(\mu)\right| B_{q}\right\rangle+\mathcal{C}_{1, \mathrm{oct}}^{\mathrm{eff}}(\mu)\left\langle J / \psi(\lambda) V(\lambda)\left|\tilde{Q}_{1, \mathrm{oct}}^{c}(\mu)\right| B_{q}\right\rangle\right. \\
& \left.+\mathcal{C}_{5}^{\mathrm{eff}}(\mu)\left\langle J / \psi(\lambda) V(\lambda)\left|Q_{5}^{c}(\mu)\right| B_{q}\right\rangle+\mathcal{C}_{5, \text { oct }}^{\mathrm{eff}}(\mu)\left\langle J / \psi(\lambda) V(\lambda)\left|Q_{5, \text { oct }}^{c}(\mu)\right| B_{q}\right\rangle\right],
\end{aligned}
$$

where $\lambda$ denotes the helicities of the final-state vector mesons and the "effective" Wilson coefficient functions are given by

$$
\begin{aligned}
& \mathcal{C}_{1}^{\mathrm{eff}}(\mu) \equiv C_{1}(\mu)+\frac{1}{3} C_{2}(\mu)+C_{3}(\mu)+\frac{1}{3} C_{4}(\mu)+C_{9}(\mu)+\frac{1}{3} C_{10}(\mu) \\
& \mathcal{C}_{1, \mathrm{oct}}^{\mathrm{eff}}(\mu) \equiv 2\left[C_{2}(\mu)+C_{4}(\mu)+C_{10}(\mu)\right] \\
& \mathcal{C}_{5}^{\mathrm{eff}}(\mu) \equiv C_{5}(\mu)+\frac{1}{3} C_{6}(\mu)+C_{7}(\mu)+\frac{1}{3} C_{8}(\mu) \\
& \mathcal{C}_{5, \mathrm{oct}}^{\mathrm{eff}}(\mu) \equiv 2\left[C_{6}(\mu)+C_{8}(\mu)\right] .
\end{aligned}
$$

The $\mu$-dependence of these Wilson coefficients is cancelled by that of the hadronic matrix elements appearing in Eq. (7). In deriving the transition matrix element in Eq. (7), we have used the relations

$$
\begin{aligned}
\tilde{Q}_{2}^{c} & =\frac{1}{3} \tilde{Q}_{1}^{c}+2 \tilde{Q}_{1, \mathrm{oct}}^{c} \\
Q_{6}^{c} & =\frac{1}{3} Q_{5}^{c}+2 Q_{5, \mathrm{oct}}^{c}
\end{aligned}
$$

with

$$
\begin{aligned}
\tilde{Q}_{1}^{c} & =\left(\bar{c}_{\alpha} c_{\alpha}\right)_{\mathrm{V}-\mathrm{A}}\left(\bar{b}_{\beta} s_{\beta}\right)_{\mathrm{V}-\mathrm{A}} \\
\tilde{Q}_{1, \mathrm{oct}}^{c} & =\left(\bar{c}_{\alpha} T_{\alpha \beta}^{a} c_{\beta}\right)_{\mathrm{V}-\mathrm{A}}\left(\bar{b}_{\gamma} T_{\gamma \delta}^{a} s_{\delta}\right)_{\mathrm{V}-\mathrm{A}}
\end{aligned}
$$

and

$$
\begin{aligned}
Q_{5}^{c} & =\left(\bar{c}_{\alpha} c_{\alpha}\right)_{\mathrm{V}+\mathrm{A}}\left(\bar{b}_{\beta} s_{\beta}\right)_{\mathrm{V}-\mathrm{A}} \\
Q_{5, \text { oct }}^{c} & =\left(\bar{c}_{\alpha} T_{\alpha \beta}^{a} c_{\beta}\right)_{\mathrm{V}+\mathrm{A}}\left(\bar{b}_{\gamma} T_{\gamma \delta}^{a} s_{\delta}\right)_{\mathrm{V}-\mathrm{A}} .
\end{aligned}
$$

Here the $3 \times 3$ matrices $T^{a}$ are the $S U(3)_{\mathrm{C}}$ generators, normalized to $\operatorname{tr}\left(T^{a} T^{b}\right)=\delta^{a b} / 2$. As we will see below, the form of the Fierz-transformed operators given above is better 
suited to analyse the $B_{q} \rightarrow J / \psi V$ decays since the $J / \psi$ is related to the $(\bar{c} c)$ pieces. The penguin contributions to $\mathcal{C}_{1}^{\text {eff }}(\mu)$ and $\mathcal{C}_{1, \text { oct }}^{\text {eff }}(\mu)$ are at most $\mathcal{O}(10 \%)$ and $\mathcal{O}(1 \%)$, respectively, as can be estimated from the values of their Wilson coefficients [27].

If one assumes that $J / \psi$ emerges from the vector parts of the $(\bar{c} c)_{V \pm A}$ quark currents appearing in the operators in Eqs. (14)-(17), the matrix elements of $\tilde{Q}_{1(\text { oct })}$ and $\tilde{Q}_{5(\text { oct })}$ will be equal and the decay amplitude Eq. (7) can be simplified considerably. Moreover, within the framework of naive factorization, we obtain (analogous for $Q_{5}^{c}$ and $Q_{5, \text { oct }}^{c}$ ):

$$
\begin{aligned}
\left\langle J / \psi(\lambda) V(\lambda)\left|\tilde{Q}_{1}^{c}\right| B_{q}\right\rangle_{\mathrm{f}} & =\left\langle J / \psi(\lambda)\left|(\bar{c} c)_{\mathrm{V}-\mathrm{A}}\right| 0\right\rangle\left\langle V(\lambda)\left|(\bar{b} s)_{\mathrm{V}-\mathrm{A}}\right| B_{s}\right\rangle \\
\left\langle J / \psi(\lambda) V(\lambda)\left|\tilde{Q}_{1, \mathrm{oct}}^{c}\right| B_{q}\right\rangle_{\mathrm{f}} & =\left\langle J / \psi(\lambda)\left|\left(\bar{c} T^{a} c\right)_{\mathrm{V}-\mathrm{A}}\right| 0\right\rangle\left\langle V(\lambda)\left|\left(\bar{b} T^{a} s\right)_{\mathrm{V}-\mathrm{A}}\right| B_{s}\right\rangle,
\end{aligned}
$$

where summation over colour-indices is understood implicitly. Consequently, since $J / \psi$ is a colour-singlet state, the factorized hadronic matrix elements of the colour-octet operators given in Eq. (19) vanish.

\section{II-C Colour-allowed decays}

In the case of the colour-allowed decays $B_{q} \rightarrow D_{s}^{*+} \bar{D}_{q}^{*}(q \in\{u, d, s\})$, the transition amplitude can be written in a way that is completely analogous to Eq. (7):

$$
\begin{aligned}
& \left\langle D_{s}^{*+}(\lambda) \bar{D}_{q}^{*}(\lambda)\left|\mathcal{H}_{\mathrm{eff}}\right| B_{q}\right\rangle=\frac{G_{\mathrm{F}}}{\sqrt{2}} V_{c s} V_{c b}^{*} \\
& \quad \times\left[\mathcal{C}_{2}^{\mathrm{eff}}(\mu)\left\langle D_{s}^{*+}(\lambda) \bar{D}_{q}^{*}(\lambda)\left|Q_{2}^{c}(\mu)\right| B_{q}\right\rangle+\mathcal{C}_{2, \mathrm{oct}}^{\mathrm{eff}}(\mu)\left\langle D_{s}^{*+}(\lambda) \bar{D}_{q}^{*}(\lambda)\left|Q_{2, \mathrm{oct}}^{c}(\mu)\right| B_{q}\right\rangle\right. \\
& \left.+\mathcal{C}_{6}^{\mathrm{eff}}(\mu)\left\langle D_{s}^{*+}(\lambda) \bar{D}_{q}^{*}(\lambda)\left|\tilde{Q}_{6}^{c}(\mu)\right| B_{q}\right\rangle+\mathcal{C}_{6, \mathrm{oct}}^{\mathrm{eff}}(\mu)\left\langle D_{s}^{*+}(\lambda) \bar{D}_{q}^{*}(\lambda)\left|\tilde{Q}_{6, \text { oct }}^{c}(\mu)\right| B_{q}\right\rangle\right] .
\end{aligned}
$$

The corresponding effective Wilson coefficient functions are, however, very different:

$$
\begin{aligned}
\mathcal{C}_{2}^{\mathrm{eff}}(\mu) & \equiv \frac{1}{3} C_{1}(\mu)+C_{2}(\mu)+\frac{1}{3} C_{3}(\mu)+C_{4}(\mu)+\frac{1}{3} C_{9}(\mu)+C_{10}(\mu) \\
\mathcal{C}_{2, \mathrm{oct}}^{\mathrm{eff}}(\mu) & \equiv 2\left[C_{1}(\mu)+C_{3}(\mu)+C_{9}(\mu)\right] \\
\mathcal{C}_{6}^{\mathrm{eff}}(\mu) & \equiv \frac{1}{3} C_{5}(\mu)+C_{6}(\mu)+\frac{1}{3} C_{7}(\mu)+C_{8}(\mu) \\
\mathcal{C}_{6, \text { oct }}^{\mathrm{eff}}(\mu) & \equiv 2\left[C_{5}(\mu)+C_{7}(\mu)\right] .
\end{aligned}
$$

In deriving Eq. (20), we have used the relations

$$
\begin{aligned}
& Q_{1}^{c}=\frac{1}{3} Q_{2}^{c}+2 Q_{2, \mathrm{oct}}^{c} \\
& \tilde{Q}_{5}^{c}=\frac{1}{3} \tilde{Q}_{6}^{c}+2 \tilde{Q}_{6, \mathrm{oct}}^{c}
\end{aligned}
$$


with

$$
\begin{aligned}
Q_{2}^{c} & =\left(\bar{c}_{\alpha} s_{\alpha}\right)_{\mathrm{V}-\mathrm{A}}\left(\bar{b}_{\beta} c_{\beta}\right)_{\mathrm{V}-\mathrm{A}} \\
Q_{2, \mathrm{oct}}^{c} & =\left(\bar{c}_{\alpha} T_{\alpha \beta}^{a} s_{\beta}\right)_{\mathrm{V}-\mathrm{A}}\left(\bar{b}_{\gamma} T_{\gamma \delta}^{a} c_{\delta}\right)_{\mathrm{V}-\mathrm{A}}
\end{aligned}
$$

and

$$
\begin{aligned}
\tilde{Q}_{6}^{c} & =-2\left(\bar{c}_{\alpha} L s_{\alpha}\right)\left(\bar{b}_{\beta} R c_{\beta}\right) \\
\tilde{Q}_{6, \text { oct }}^{c} & =-2\left(\bar{c}_{\alpha} L T_{\alpha \beta}^{a} s_{\beta}\right)\left(\bar{b}_{\gamma} R T_{\gamma \delta}^{a} c_{\delta}\right) .
\end{aligned}
$$

Here $L$ and $R$ correspond to the Dirac structures $\hat{1}-\gamma_{5}$ and $\hat{1}+\gamma_{5}$, respectively. The $D_{s}^{*+}$ meson emerges from the $(\bar{c} s)$ pieces of these operators. Since it is a vector meson, we have

$$
\left\langle D_{s}^{*+}\left|\bar{c}_{\alpha} L s_{\alpha}\right| 0\right\rangle=0
$$

and hence the factorized matrix element of $\tilde{Q}_{6}^{c}$ vanishes. As in Sec. [1-B, the hadronic matrix elements of the colour-octet operators vanish within the factorization approximation because of their colour-structure.

\section{II-D Observables of the angular distributions}

The hadronic matrix element of a generic four-quark operator $\mathcal{Q}$ between the state vectors $\left\langle V_{1}(\lambda) V_{2}(\lambda)\right|$ and $\left|B_{q}\right\rangle$ has the following general Lorentz-decomposition [5, 6]:

$$
\begin{aligned}
& \left\langle V_{1}(\lambda) V_{2}(\lambda)|\mathcal{Q}| B_{q}\right\rangle= \\
& \epsilon_{V_{1}, \mu}(\lambda)^{*} \epsilon_{V_{2}, \nu}(\lambda)^{*}\left[a g^{\mu \nu}+\frac{b}{m_{V_{1}} m_{V_{2}}} p_{V_{2}}^{\mu} p_{V_{1}}^{\nu}+i \frac{c}{m_{V_{1}} m_{V_{2}}} \varepsilon^{\mu \nu \alpha \beta} p_{V_{1}, \alpha} p_{V_{2}, \beta}\right],
\end{aligned}
$$

where the symbols $\epsilon(\lambda)$ denote the polarization vectors of the final-state vector mesons $V_{1}$ and $V_{2}$. A similar parametrization can be employed to express the transition matrix elements [Eqs. (17) and (20)], yielding

$$
\begin{aligned}
a & =\frac{G_{\mathrm{F}}}{\sqrt{2}} V_{c s} V_{c b}^{*}\left[\mathcal{C}_{i}^{\mathrm{eff}}(\mu) A_{i}^{\mathrm{f}}+\mathcal{C}_{i+4}^{\mathrm{eff}}(\mu) A_{i+4}^{\mathrm{f}}\right. \\
& \left.+\mathcal{C}_{i}^{\mathrm{eff}}(\mu) A_{i}^{\mathrm{nf}}(\mu)+\mathcal{C}_{i, \mathrm{oct}}^{\mathrm{eff}}(\mu) A_{i, \mathrm{oct}}^{\mathrm{nf}}(\mu)+\mathcal{C}_{i+4}^{\mathrm{eff}}(\mu) A_{i+4}^{\mathrm{nf}}(\mu)+\mathcal{C}_{i+4, \mathrm{oct}}^{\mathrm{eff}}(\mu) A_{i+4, \mathrm{oct}}^{\mathrm{nf}}(\mu)\right] \\
b & =\frac{G_{\mathrm{F}}}{\sqrt{2}} V_{c s} V_{c b}^{*}\left[\mathcal{C}_{i}^{\mathrm{eff}}(\mu) B_{i}^{\mathrm{f}}+\mathcal{C}_{i+4}^{\mathrm{eff}}(\mu) B_{i+4}^{\mathrm{f}}\right. \\
& \left.+\mathcal{C}_{i}^{\mathrm{eff}}(\mu) B_{i}^{\mathrm{nf}}(\mu)+\mathcal{C}_{i, \mathrm{oct}}^{\mathrm{eff}}(\mu) B_{i, \mathrm{oct}}^{\mathrm{nf}}(\mu)+\mathcal{C}_{i+4}^{\mathrm{eff}}(\mu) B_{i+4}^{\mathrm{nf}}(\mu)+\mathcal{C}_{i+4, \mathrm{oct}}^{\mathrm{eff}}(\mu) B_{i+4, \mathrm{oct}}^{\mathrm{nf}}(\mu)\right]
\end{aligned}
$$




$$
\begin{aligned}
c & =\frac{G_{\mathrm{F}}}{\sqrt{2}} V_{c s} V_{c b}^{*}\left[\mathcal{C}_{i}^{\mathrm{eff}}(\mu) C_{i}^{\mathrm{f}}+\mathcal{C}_{i+4}^{\mathrm{eff}}(\mu) C_{i+4}^{\mathrm{f}}\right. \\
& \left.+\mathcal{C}_{i}^{\mathrm{eff}}(\mu) C_{i}^{\mathrm{nf}}(\mu)+\mathcal{C}_{i, \mathrm{oct}}^{\mathrm{eff}}(\mu) C_{i, \mathrm{oct}}^{\mathrm{nf}}(\mu)+\mathcal{C}_{i+4}^{\mathrm{eff}}(\mu) C_{i+4}^{\mathrm{nf}}(\mu)+\mathcal{C}_{i+4, \mathrm{oct}}^{\mathrm{eff}}(\mu) C_{i+4, \mathrm{oct}}^{\mathrm{nf}}(\mu)\right],
\end{aligned}
$$

where the index $i$ distinguishes between colour-suppressed $(i=1)$ and colour-allowed $(i=2)$ decays and "f" and "nf" correspond to "factorized" and "non-factorized" matrix elements, respectively. Note that the factorized amplitudes do not depend on the renormalization scale $\mu$. Since the Wilson coefficients depend on this scale, this already signals the need for non-factorizable contributions to cancel the $\mu$-dependence in Eqs. (33)-(35) (see e.g. Ref. [32] for a further discussion of that point).

In the following sections we will analyse the decays $B_{q} \rightarrow V_{1} V_{2}$ in terms of linear polarization states. The corresponding decay amplitudes take the form [23, 33]

$$
A\left(B_{q}(t) \rightarrow V_{1} V_{2}\right)=\frac{A_{0}(t)}{x} \epsilon_{V_{1}}^{* L} \epsilon_{V_{2}}^{* L}-A_{\|}(t) \epsilon_{V_{1}}^{* T} \cdot \epsilon_{V_{2}}^{* T} / \sqrt{2}-i A_{\perp}(t) \epsilon_{V_{1}}^{*} \times \epsilon_{V_{2}}^{*} \cdot \hat{\mathbf{p}}_{V_{2}} / \sqrt{2},
$$

where $x \equiv p_{V_{1}} \cdot p_{V_{2}} /\left(m_{V_{1}} m_{V_{2}}\right)$ and $\hat{\mathbf{p}}_{V_{2}}$ is the unit vector along the direction of motion of $V_{2}$ in the rest frame of $V_{1}$. Here the time dependences originate from $B_{q}-\bar{B}_{q}$ mixing. In our notation, an unmixed $B_{q}$ meson is present at $t=0$.

The linear polarization amplitudes at $t=0$ defined by Eq. (36) can be expressed in terms of $a, b$ and $c$ as follows [33]:

$$
\begin{aligned}
& A_{0}(0)=-x a-\left(x^{2}-1\right) b \\
& A_{\|}(0)=\sqrt{2} a \\
& A_{\perp}(0)=\sqrt{2\left(x^{2}-1\right)} c .
\end{aligned}
$$

At time $t=0$, the angular distributions for $B_{q} \rightarrow V_{1} V_{2}$ depend on the observables $\left|A_{0}(0)\right|,\left|A_{\|}(0)\right|,\left|A_{\perp}(0)\right|$ and on the two phases $\delta_{1} \equiv \operatorname{Arg}\left[A_{\|}(0)^{*} A_{\perp}(0)\right]$ and $\delta_{2} \equiv$ $\operatorname{Arg}\left[A_{0}(0)^{*} A_{\perp}(0)\right]$, which are $\mathrm{CP}$-conserving strong phases that are $0(\bmod \pi)$ in the absence of final-state interactions (probably not a justifiable assumption for the coloursuppressed modes). Quantitative estimates for these observables will be given in the following subsection.

\section{II-E Factorization tests and estimates of observables}

While the non-factorizable contributions to $a, b$ and $c$ cannot be calculated at present, the evaluation of the factorizable contributions is straightforward. Without yet going into the details of which form factors to employ, the naive factorization assumption yields many testable consequences. For example, time-reversal invariance forces the form factors parametrizing quark currents to be all relatively real. Consequently, naive factorization predicts the same strong phase $(\bmod \pi)$ for the three amplitudes $A_{0}(0), A_{\|}(0), A_{\perp}(0)$. It therefore predicts vanishing values of the two observables [17, 19, 20

$$
\operatorname{Im}\left[A_{0}^{*}(0) A_{\perp}(0)\right]=0
$$




$$
\operatorname{Im}\left[A_{\|}^{*}(0) A_{\perp}(0)\right]=0
$$

and the equality

$$
\operatorname{Re}\left[A_{0}^{*}(0) A_{\|}(0)\right]= \pm\left|A_{0}(0) A_{\|}(0)\right| .
$$

The breakdown of the naive factorization assumption is unequivocally proved if any of the three equations (38)-(40) is not satisfied. Detailed comparisons of polarization amplitudes in non-leptonic and semi-leptonic decays test additional implications of the naive factorization assumption. The phenomenology of detailed studies of the full non-trivial angular distributions is thus much richer than the single factorization test available for a pseudoscalar decaying into two pseudoscalars [18. While the above equations represent general tests of the factorization assumption, it is also useful to examine the predictions for the observables of the angular distributions for various form factor ansätze.

\section{II-E.1 The colour-suppressed decays $B_{q} \rightarrow J / \psi V$}

The factorized amplitudes for $B_{q} \rightarrow J / \psi V$ with $(q, V) \in\left\{(s, \phi) ;\left(d, K^{* 0}\right) ;\left(u, K^{*+}\right)\right\}$ are given by [16, 21, 22]:

$$
\begin{aligned}
& A_{1}^{\mathrm{f}}=-f_{J / \psi} m_{J / \psi}\left(m_{B_{q}}+m_{V}\right) A_{1}^{B_{q} V}\left(m_{J / \psi}^{2}\right)=A_{5}^{\mathrm{f}} \\
& B_{1}^{\mathrm{f}}=2 \frac{f_{J / \psi} m_{J / \psi}^{2} m_{V}}{m_{B_{q}}+m_{V}} A_{2}^{B_{q} V}\left(m_{J / \psi}^{2}\right)=B_{5}^{\mathrm{f}} \\
& C_{1}^{\mathrm{f}}=2 \frac{f_{J / \psi} m_{J / \psi}^{2} m_{V}}{m_{B_{q}}+m_{V}} V^{B_{q} V}\left(m_{J / \psi}^{2}\right)=C_{5}^{\mathrm{f}},
\end{aligned}
$$

where we have used the notation of Bauer, Stech and Wirbel for the form factors $A_{i}^{B_{q} V}\left(q^{2}\right)$ and $V^{B_{q} V}\left(q^{2}\right)$ of quark currents [16]. The parameter $f_{J / \psi}$ denotes the $J / \psi$ decay constant, which can be determined from the $J / \psi \rightarrow e^{+} e^{-}$rate, yielding $f_{J / \psi}=395 \mathrm{MeV}$.

At present, several methods for obtaining the form factors $A_{1}\left(m_{J / \psi}^{2}\right), A_{2}\left(m_{J / \psi}^{2}\right)$ and $V\left(m_{J / \psi}^{2}\right)$ for the $B \rightarrow K^{*}$ case are on the market. Using $S U(3)$ flavour symmetry of strong interactions, the $B \rightarrow K^{*}$ form factors can be related to the $B_{s} \rightarrow \phi$ case. In Table 11 we have collected the form factors proposed by several authors [16, 34, 35], and have moreover given the corresponding predictions for the ratios of observables of the angular distributions. These ratios should suffer less from unknown $S U(3)$-breaking corrections than the observables themselves. Note that these ratios are independent of the Wilson coefficients within the factorization approach.

The quantity

$$
\frac{\Gamma_{0}(0)}{\Gamma_{0}(0)+\Gamma_{T}(0)} \equiv \frac{\left|A_{0}(0)\right|^{2}}{\left|A_{0}(0)\right|^{2}+\left|A_{\|}(0)\right|^{2}+\left|A_{\perp}(0)\right|^{2}}
$$

describes the ratio of the longitudinal to the total rate at $t=0$. Although CDF 36] claims to have measured this quantity, from their untagged data sample, to be $0.56 \pm$ 


\begin{tabular}{|c|c|c|c|}
\hline Observable & BSW [16] & Soares [34] & Cheng [35] \\
\hline$A_{1}^{B K^{*}}\left(m_{J / \psi}^{2}\right)$ & 0.46 & 0.42 & 0.41 \\
\hline$A_{2}^{B K^{*}}\left(m_{J / \psi}^{2}\right)$ & 0.46 & 0.43 & 0.36 \\
\hline$V^{B K^{*}}\left(m_{J / \psi}^{2}\right)$ & 0.55 & 1.08 & 0.72 \\
\hline$\left|A_{\|}(0)\right| /\left|A_{0}(0)\right|$ & $0.81(0.77)$ & $0.82(0.78)$ & $0.75(0.70)$ \\
\hline$\left|A_{\perp}(0)\right| /\left|A_{0}(0)\right|$ & $0.41(0.40)$ & $0.89(0.88)$ & $0.55(0.54)$ \\
\hline$\Gamma_{0}(0) /\left(\Gamma_{0}(0)+\Gamma_{T}(0)\right)$ & $0.55(0.57)$ & $0.40(0.42)$ & $0.54(0.56)$ \\
\hline$\delta_{1}$ & $\pi$ & $\pi$ & $\pi$ \\
\hline$\delta_{2}$ & 0 & 0 & 0 \\
\hline
\end{tabular}

Table 1: Predictions for form factors and $B_{s} \rightarrow J / \psi \phi\left(B \rightarrow J / \psi K^{*}\right)$ observables.

0.21 (stat.) ${ }_{-0.04}^{+0.02}$ (syst.), their claim is valid only if the CP-odd component of $B_{s} \rightarrow J / \psi \phi$ is negligible, or if the lifetime difference $\Delta \Gamma$ can be ignored.

The $2^{\text {nd }}-4^{\text {th }}$ columns of Table 11 are calculated within the framework of naive factorization, i.e. we have inserted Eq. (41) into Eqs. (33)-(35) and have omitted the "nf" terms in order to calculate the amplitudes in Eq. (37).

The form factors given by Soares [34] are obtained from $\bar{D} \rightarrow K^{(*)} l \bar{\nu}_{l}$ data by using heavy-quark symmety relations [37] and assuming the monopole momentum-transfer dependence of the BSW model [16]. Some more form-factor models and their predictions are discussed in [38]. Note that the small difference between the $B_{s} \rightarrow J / \psi \phi$ and $B \rightarrow J / \psi K^{*}$ results in Table 1 is related to phase-space effects and not to any $S U(3)$ breaking effects in the corresponding hadronic matrix elements.

Looking at Table 1 , we observe that the "factorized" predictions for $\left|A_{\|}(0)\right| /\left|A_{0}(0)\right|$ are rather stable $(\approx 0.8)$, while $\left|A_{\perp}(0)\right| /\left|A_{0}(0)\right|$ depends strongly on the method used for obtaining the form factors. A common feature of all results is $\delta_{1}=\pi$ and $\delta_{2}=0$. Therefore a measurement of non-trivial phases $\delta_{1}$ and $\delta_{2}$ would imply the presence of strong final-state interactions and non-factorizable contributions.

Whereas the use of the factorization assumption is very questionable in the case of the channels $B_{s} \rightarrow J / \psi \phi$ and $B \rightarrow J / \psi K^{*}$, flavour $S U(3)$ symmetry is probably a good working assumption. Thus all the hadronization dynamics of the $B_{s} \rightarrow J / \psi \phi$ decay, such as the phases $\delta_{1}$ and $\delta_{2}$ and magnitudes of the amplitudes

$$
A_{0}(0), A_{\|}(0), A_{\perp}(0),
$$

can be obtained from the $B \rightarrow J / \psi K^{*}$ modes. This approach may be helpful to extract the CKM phase $\phi_{\text {CKM }}$ (see Eq. (1)), as we will see below.

${ }^{1}$ Although those $S U(3)$ relations are mostly trivial, one subtlety due to quantum-coherence must be emphasized. Because of the $S U(3)$ relations in the unmixed amplitudes

$$
A_{f}\left(B_{s} \rightarrow J / \psi \phi\right)=A_{f}\left(B \rightarrow J / \psi K^{*}\right), \quad \text { where } f=0, \|, \perp \quad,
$$

the magnitudes of the amplitudes for $B_{s}^{L}$ or $B_{s}^{H}$ decays into CP-even or CP-odd $J / \psi \phi$ final-state 
The factorization assumption should work much better for the transitions $B_{s} \rightarrow$ $D_{s}^{*+} D_{s}^{*-}$ and $B \rightarrow D_{s}^{*+} \bar{D}^{*}$. Therefore the results presented in the following subsection should be more reliable than those summarized in Table 1 .

\section{II-E.2 The colour-allowed decays $B_{q} \rightarrow D_{s}^{*+} \bar{D}_{q}^{*}$}

Using again the same notation as Ref. [16], we get the following "factorized" results for the modes $B_{q} \rightarrow D_{s}^{*+} \bar{D}_{q}^{*}(q \in\{u, d, s\})$ [23, 24, 26]:

$$
\begin{array}{ll}
A_{2}^{\mathrm{f}}=-f_{D_{s}^{*}} m_{D_{s}^{*}}\left(m_{B_{q}}+m_{D_{q}^{*}}\right) A_{1}^{B_{q} D_{q}^{*}}\left(m_{D_{s}^{*}}^{2}\right), & A_{6}^{\mathrm{f}}=0 \\
B_{2}^{\mathrm{f}}=2 \frac{f_{D_{s}^{*}} m_{D_{s}^{*}}^{2} m_{D_{q}^{*}}}{m_{B_{q}}+m_{D_{q}^{*}}} A_{2}^{B_{q} D_{q}^{*}}\left(m_{D_{s}^{*}}^{2}\right), & B_{6}^{\mathrm{f}}=0 \\
C_{2}^{\mathrm{f}}=2 \frac{f_{D_{s}^{*}} m_{D_{s}^{*}}^{2} m_{D_{q}^{*}}}{m_{B_{q}}+m_{D_{q}^{*}}} V^{B_{q} D_{q}^{*}}\left(m_{D_{s}^{*}}^{2}\right), & C_{6}^{\mathrm{f}}=0 .
\end{array}
$$

The parameter $f_{D_{s}^{*}}$ is the $D_{s}^{*}$ decay constant. The spin symmetry of HQET implies $f_{D_{s}^{*}} \approx$ $f_{D_{s}}$. A recent compilation of measurements of $f_{D_{s}}$ from $D_{s} \rightarrow \mu \bar{\nu}$ gives $(241 \pm 21 \pm 30)$ $\mathrm{MeV}$ [39].

In the case of $B_{q} \rightarrow \bar{D}_{q}^{*}$ transitions we have rather tight restrictions from HQET (for reviews, see for example [25]) for the corresponding form factors. The following ratios turn out to be useful to implement these HQET constraints [40]:

$$
\begin{aligned}
& R_{1}(w)=\left[1-\frac{q^{2}}{\left(m_{B_{q}}+m_{D_{q}^{*}}\right)^{2}}\right] \frac{V^{B D^{*}}\left(q^{2}\right)}{A_{1}^{B D^{*}}\left(q^{2}\right)} \\
& R_{2}(w)=\left[1-\frac{q^{2}}{\left(m_{B_{q}}+m_{D_{q}^{*}}\right)^{2}}\right] \frac{A_{2}^{B D^{*}}\left(q^{2}\right)}{A_{1}^{B D^{*}}\left(q^{2}\right)},
\end{aligned}
$$

where $R_{1}(w)$ and $R_{2}(w)$ are defined in such a way that we have

$$
R_{1}(w)=R_{2}(w)=1
$$

for all values of $w$ in the strict heavy-quark limit. The kinematical variable $w$ is defined by

$$
w=\frac{m_{B_{q}}^{2}+m_{D_{q}^{*}}^{2}-q^{2}}{2 m_{B_{q}} m_{D_{q}^{*}}} .
$$

configurations, respectively, are a factor of $\sqrt{2}$ larger than their corresponding $B \rightarrow J / \psi K^{*}$ ones. [Here the $K^{*}$ is seen in a flavour-specific mode. If $K^{*}$ is neutral and is observed as $\pi^{0} K_{S}$, quantum coherence in $B^{0}-\overline{B^{0}}$ must also be taken into account.] If the CP-even processes dominate, then

$$
\Gamma\left(B_{s}^{L} \rightarrow J / \psi \phi\right) \approx 2 \Gamma\left(B \rightarrow J / \psi K^{*}\right) .
$$

Studies of $B_{s}$ versus $B$ production fractions can thus be undertaken, since the lifetimes will be precisely known. 


\begin{tabular}{|c|c|c|c|}
\hline Observable & BSW & HQET $_{\text {strict }}$ & HQET $_{\text {sym...break. }}$ \\
\hline$A_{1}^{B D^{*}}\left(m_{D_{s}^{*}}^{2}\right)$ & 0.72 & $0.70(0.68)$ & $0.70(0.68)$ \\
\hline$A_{2}^{B D^{*}}\left(m_{D_{s}^{*}}^{2}\right)$ & 0.76 & $0.76(0.75)$ & $0.54(0.53)$ \\
\hline$V^{B D^{*}}\left(m_{D_{s}^{*}}^{2}\right)$ & 0.79 & $0.76(0.75)$ & $0.89(0.88)$ \\
\hline$\left|A_{\|}(0)\right| /\left|A_{0}(0)\right|$ & $0.90(0.90)$ & $0.91(0.91)$ & $0.81(0.80)$ \\
\hline$\left|A_{\perp}(0)\right| /\left|A_{0}(0)\right|$ & $0.32(0.32)$ & $0.32(0.33)$ & $0.33(0.34)$ \\
\hline$\Gamma_{0}(0) /\left(\Gamma_{0}(0)+\Gamma_{T}(0)\right)$ & $0.52(0.52)$ & $0.52(0.52)$ & $0.57(0.57)$ \\
\hline$\delta_{1}$ & $\pi$ & $\pi$ & $\pi$ \\
\hline$\delta_{2}$ & 0 & 0 & 0 \\
\hline
\end{tabular}

Table 2: Predictions for form factors and $B_{s} \rightarrow D_{s}^{*+} D_{s}^{*-}\left(B \rightarrow D_{s}^{*+} \bar{D}^{*}\right)$ observables.

The value of the momentum transfer $q^{2}$ relevant for Eq. (44) is $q^{2}=m_{D_{s}^{*}}^{2}$. The form factor $A_{1}^{B D^{*}}\left(q^{2}\right)$ is usually written as

$$
A_{1}^{B D^{*}}\left(q^{2}\right)=\frac{m_{B_{q}}+m_{D_{q}^{*}}}{2 \sqrt{m_{B_{q}} m_{D_{q}^{*}}}}\left[1-\frac{q^{2}}{\left(m_{B_{q}}+m_{D_{q}^{*}}\right)^{2}}\right] h_{A_{1}}(w),
$$

where $h_{A_{1}}(w)$ corresponds to the Isgur-Wise function in the strict heavy-quark limit and can be written as

$$
h_{A_{1}}(w)=\mathcal{F}(1)\left[1-\rho_{A_{1}}^{2}(w-1)+\mathcal{O}\left((w-1)^{2}\right)\right] .
$$

The current status of the normalization $\mathcal{F}(1)$ and of the "slope parameter" $\rho_{A_{1}}^{2}$ has been summarized recently by Neubert in Ref. [40]. The form factor $A_{1}^{B D^{*}}\left(q^{2}\right)$ is protected by Luke's theorem [41] against $1 / m_{Q}$ corrections at zero recoil. The other form factors $A_{2}^{B D^{*}}\left(q^{2}\right)$ and $V^{B D^{*}}\left(q^{2}\right)$ are not protected by this theorem. From calculations based on HQET one expects a rather weak dependence of $R_{1}(w)$ and $R_{2}(w)$ on $w$ and therefore uses

$$
\begin{aligned}
& R_{1}(w)=R_{1}[1+\mathcal{O}(w-1)] \\
& R_{2}(w)=R_{2}[1+\mathcal{O}(w-1)] .
\end{aligned}
$$

In our analysis we will neglect the $w$-dependence completely.

Following these lines we have calculated the results for the form factors and ratios of observables, which should receive smaller $S U(3)$-breaking corrections than the observables themselves, summarized in Table 2. For completeness we have also given the results obtained by applying the BSW model [16] in the $2^{\text {nd }}$ column. In order to calculate the $3^{\text {rd }}$ and $4^{\text {th }}$ columns, we have used $\mathcal{F}(1)=0.91$ and $\rho_{A_{1}}^{2}=0.91$ 40. The columns denoted by $\mathrm{HQET}_{\text {strict }}$ and $\mathrm{HQET}_{\text {sym.-break. }}$ correspond to $R_{1}=R_{2}=1$ and $R_{1}=1.18$, $R_{2}=0.71$, respectively, where we have employed the results by Neubert [40] to take into 
account HQET symmetry-breaking corrections. Within the factorization approximation we obtain the following simple expressions for $A_{\|}(0) / A_{0}(0)$ and $A_{\perp}(0) / A_{0}(0)$ in terms of the HQET parameters:

$$
\begin{aligned}
& \frac{A_{\|}(0)}{A_{0}(0)}=\sqrt{2}\left[\frac{m_{D_{s}^{*}}}{m_{B_{q}}}\left(\frac{x^{2}-1}{w+1}\right) R_{2}(w)-x\right]^{-1} \\
& \frac{A_{\perp}(0)}{A_{0}(0)}=-\left[\frac{m_{D_{s}^{*}}}{m_{B_{q}}}\left(\frac{\sqrt{x^{2}-1}}{w+1}\right) R_{1}(w)\right] \frac{A_{\|}(0)}{A_{0}(0)}
\end{aligned}
$$

where the kinematical variable

$$
x=\frac{m_{B_{q}}^{2}-m_{D_{q}^{*}}^{2}-m_{D_{s}^{*}}^{2}}{2 m_{D_{q}^{*}} m_{D_{s}^{*}}}
$$

has been defined after Eq. (36).

If we compare Table 8 with Table 1, we note that the results for the observables depend much less on the way of obtaining the form factors. Also the "old" BSW model is in rather good agreement with the HQET predictions, which is quite remarkable. Therefore the results given in Table 2 are more reliable than those collected in Table 1. In this respect it is also important to note that the non-factorizable contributions appearing in Eqs. (33)-(35) should play a minor role for the colour-allowed decay class and that $\delta_{1}=\pi$ and $\delta_{2}=0$ is expected to hold on rather solid ground. Because of the latter feature, CP-violating effects arising in untagged $B_{s} \rightarrow D_{s}^{*+} D_{s}^{*-}$ data samples should be a promising way to extract the weak phase $\phi_{\mathrm{CKM}}$ (see Eq. (II)), as has been outlined in detail in Ref. [8].

\section{The angular-moment analysis}

The main focus of this section is the efficient determination of the observables discussed in Sect. [I-D and [I-E. This can be accomplished by an angular-moment analysis [1]. In this approach, the observed data are weighted by judiciously chosen functions, which project out any desired observable. Whereas Ref. [1] determines the moments for a few choice angular distributions, using spherical harmonics, this paper indicates how to determine suitable weighting functions for all kinds of angular distributions, using only orthogonality arguments (without invoking spherical harmonics).

Let us denote the angular distribution of a given decay by

$$
f(\Theta, \alpha ; t)=\sum_{i} b^{(i)}(\alpha ; t) g^{(i)}(\Theta)
$$

where $\alpha$ represents all the parameters that are independent of the kinematics, which is described by certain decay angles. In general, the physical process involves an arbitrary 
number of such angles denoted generically by $\Theta$. For the examples considered in this article, $i$ runs from 1 to 6 , and we have

$$
\alpha=\left\{\Gamma_{H}, \Gamma_{L}, \Delta m,\left|A_{0}(0)\right|,\left|A_{\|}(0)\right|,\left|A_{\perp}(0)\right|, \delta_{1}, \delta_{2}, \phi_{\mathrm{CKM}}\right\} .
$$

All the quantities of interest are encoded in the time evolution of the observables $b^{(i)}(\alpha ; t)$. In the following discussion the $\alpha$ - and $t$-dependence of the $b^{(i)}$ 's is implicit wherever not explicitly stated.

The usual method for extracting $b^{(i)}$ 's is to use an unbinned maximum likelihood fit [4]. Performing such a fit for a given quantity requires some idea of the values of the other quantities. When one deals with limited statistics, one may want to exploit alternative methods that completely decouple the extraction of one observable from all the others. Luckily such a method exists, the angular-moment analysis.

If we can find a weighting function $w^{(i)}(\Theta)$ for each $i$ such that

$$
\int[D \Theta] w^{(i)}(\Theta) g^{(k)}(\Theta)=\delta_{i k}=\left\{\begin{array}{l}
0 \text { for } i \neq k \\
1 \text { for } i=k
\end{array}\right.
$$

then the $b^{(i)}$ 's can be obtained directly from

$$
b^{(i)}=\int[D \Theta] w^{(i)}(\Theta) f_{\operatorname{expt}}(\Theta) .
$$

Here $[D \Theta]$ denotes the appropriate measure for integrating over all angles $\Theta$, and $f_{\text {expt }}(\Theta)$ denotes the observed full angular distribution. For a small number of events $(N)$, the form of the function $f_{\text {expt }}(\Theta)$ will not be known, but only the values for $\Theta$ will be known for each event. In that case the above equation reduces to

$$
b^{(i)}=\frac{1}{N} \sum_{\text {events }} w^{(i)}(\Theta) .
$$

These $b^{(i)}$ 's can then be used directly for studying their $(\alpha ; t)$ dependence.

That $w^{(k)}$ 's can always be found for any angular distribution follows from the linear independence of the $g^{(i)}$ 's (they have to be independent for the angular distribution to be legitimate). The vector space $\mathcal{V}_{k}$ spanned by all $g^{(j)}$ 's for $j \neq k$ is a proper subspace of the vector space $\mathcal{V}$ spanned by all $g^{(i)}$ 's. Then there exists a one-dimensional vector space $\mathcal{W}$ such that $\mathcal{V}=\mathcal{V}_{k} \oplus \mathcal{W}$ and $\mathcal{V}_{k} \perp \mathcal{W}$. Here the scalar product is defined as $v_{1} \cdot v_{2} \equiv \int[D \Theta] v_{1}(\Theta) v_{2}(\Theta) ; w^{(k)}$ is then the element of $\mathcal{W}$ with proper magnitude.

For a given set of $g^{(i)}$ 's, the choice of $w^{(i)}$ 's need not be unique. We can always take any vector space $\mathcal{V}^{\prime} \supset \mathcal{V}$ and the corresponding projection space $\mathcal{W}^{\prime}$ such that $\mathcal{V}^{\prime}=\mathcal{V}_{k} \oplus \mathcal{W}^{\prime}$ and $\mathcal{V}_{k} \perp \mathcal{W}^{\prime}$. Then any $w^{(k)} \in \mathcal{W}^{\prime}$ with $w^{(k)} \cdot g^{(k)}=1$ will serve our purpose.

We now indicate an explicit procedure for finding a set of weighting functions applicable to any given angular distribution. For a theoretical angular distribution of the 
form $f=\sum_{i=1}^{n} b^{(i)} g^{(i)}$ (the dependence on angles and time is implicit),

$$
w^{(i)}=\sum_{j=1}^{n} \lambda_{i j} g^{(j)}
$$

is a proper weighting function, where the $n^{2}$ unknowns $\lambda_{i j}$ are solutions of the $n^{2}$ simultaneous equations

$$
\delta_{i k}=\sum_{j=1}^{n} \lambda_{i j} \int[D \Theta] g^{(j)} g^{(k)} .
$$

The existence of such a solution follows from the vector-space arguments given earlier. The $w^{(i)}$ 's need not be restricted to the vector space spanned by the $n$ vectors $g^{(j)}$ 's, in which case the unknowns $\lambda_{i j}$ will be underdetermined and more than one set of $w^{(i)}$ 's will serve our purpose.

It is crucial to observe that the weighting functions $w^{(i)}$ depend only on the angular terms and not on the values of the observables $b^{(j)}$. The implication is that no matter how complicated the detailed angular distribution, there always exists an angular weighting, which projects out the desired observables alone. We therefore recommend the use of moments whenever one wishes to extract observables from measured angular distributions, such as in weak decays of baryons [2] or pseudoscalars $\left[P \rightarrow V \ell \nu, X{ }_{J} \ell \nu, V V\right.$, etc.], or strong and electromagnetic decays [3]. The utility of this approach cannot be overemphasized. For instance, the moment analysis allows the study of the $q^{2}$ dependence of each of the observables separately in the process $P \rightarrow V \ell \nu$. This could prove useful for the extraction of form factors and the determination of CKM elements, e.g. $V_{c b}$ and $V_{u b}$.

We note that there exist many legitimate choices of weighting functions. The optimal choice depends on the numerical values of the observables [1] and on the detector configuration.

\section{The angular distribution of the colour-suppressed decays $B_{s} \rightarrow J / \psi \phi$ and $B \rightarrow J / \psi K^{*}$}

In this section we give the angular distribution of the decays $B_{s} \rightarrow J / \psi \phi$ and $B \rightarrow$ $J / \psi K^{*}$, their time-dependences and appropriate weighting functions.

\section{IV-A The decay $B_{s} \rightarrow J / \psi\left(\rightarrow l^{+} l^{-}\right) \phi\left(\rightarrow K^{+} K^{-}\right)$}

An analysis of this process has been performed in 33 in terms of linear polarization states of the final-state vector mesons. The corresponding decay amplitude has the same form as Eq. (36). Since the amplitudes $A_{0, \|}$ and $A_{\perp}$ are related to CP-even and CP-odd final-state configurations, respectively, they differ in time evolution as well as angular distribution. The angular distribution can be used to separate these components and their time evolution can be studied individually. 
The differential decay rate at time $t$ as a function of a generic variable $x$ will be denoted by

$$
\frac{d \Gamma(t, x)}{d x} \equiv \frac{1}{N(t)} \frac{d^{2} N(t)}{d x d t} .
$$

Consequently the normalized number of decays in the intervals $[t, t+\Delta t]$ and $[x, x+\Delta x]$ is given by

$$
\frac{d \Gamma(t, x)}{d x} \Delta x \Delta t=\frac{1}{N(t)} \frac{d^{2} N(t)}{d x d t} \Delta x \Delta t .
$$

\section{IV-B Tagged decays}

In the case of $B_{s} \rightarrow J / \psi \phi$, the three-angle distribution for the decay of an initially present (i.e. tagged) $B_{s}$ meson takes the form [33]

$$
\begin{aligned}
& \frac{d^{3} \Gamma\left[B_{s}(t) \rightarrow J / \psi\left(\rightarrow l^{+} l^{-}\right) \phi\left(\rightarrow K^{+} K^{-}\right)\right]}{d \cos \theta d \varphi d \cos \psi} \propto \frac{9}{32 \pi}\left[2\left|A_{0}(t)\right|^{2} \cos ^{2} \psi\left(1-\sin ^{2} \theta \cos ^{2} \varphi\right)\right. \\
+ & \sin ^{2} \psi\left\{\left|A_{\|}(t)\right|^{2}\left(1-\sin ^{2} \theta \sin ^{2} \varphi\right)+\left|A_{\perp}(t)\right|^{2} \sin ^{2} \theta-\operatorname{Im}\left(A_{\|}^{*}(t) A_{\perp}(t)\right) \sin 2 \theta \sin \varphi\right\} \\
+ & \left.\frac{1}{\sqrt{2}} \sin 2 \psi\left\{\operatorname{Re}\left(A_{0}^{*}(t) A_{\|}(t)\right) \sin ^{2} \theta \sin 2 \varphi+\operatorname{Im}\left(A_{0}^{*}(t) A_{\perp}(t)\right) \sin 2 \theta \cos \varphi\right\}\right] .
\end{aligned}
$$

Throughout this section we will apply the same conventions as in Ref. [33], i.e. $\phi$ moves in $x$ direction in the $J / \psi$ rest frame, the $z$ axis is perpendicular to the decay plane of $\phi \rightarrow K^{+} K^{-}$, and $p_{y}\left(K^{+}\right) \geq 0$. The coordinates $(\theta, \varphi)$ describe the decay direction of $l^{+}$ in the $J / \psi$ rest frame and $\psi$ is the angle made by $\vec{p}\left(K^{+}\right)$with the $x$ axis in the $\phi$ rest frame. With this convention,

$$
\begin{gathered}
\mathbf{x}=\mathbf{p}_{\phi}, \mathbf{y}=\frac{\mathbf{p}_{K^{+}}-\mathbf{p}_{\phi}\left(\mathbf{p}_{\phi} \cdot \mathbf{p}_{K^{+}}\right)}{\mid \mathbf{p}_{K^{+}}-\mathbf{p}_{\phi}\left(\mathbf{p}_{\phi} \cdot \mathbf{p}_{K^{+}}\right)}, \mathbf{z}=\mathbf{x} \times \mathbf{y} \\
\sin \theta \quad \cos \varphi=\mathbf{p}_{\ell^{+}} \cdot \mathbf{x}, \sin \theta \quad \sin \varphi=\mathbf{p}_{\ell^{+}} \cdot \mathbf{y}, \cos \theta=\mathbf{p}_{\ell^{+}} \cdot \mathbf{z} .
\end{gathered}
$$

Here, the bold-face characters represent unit 3-vectors and everything is measured in the rest frame of $J / \psi$. Also

$$
\cos \psi=-\mathbf{p}_{K^{+}}^{\prime} \cdot \mathbf{p}_{J / \psi}^{\prime},
$$

where the primed quantities are unit vectors measured in the rest frame of $\phi$.

The time dependence of the right-hand side of Eq. (64) can be read off from Table 3 , where $\Delta m \equiv m_{H}-m_{L}>0$ is the mass difference of the mass eigenstates $B_{s}^{H}$ and $B_{s}^{L}$ of the $B_{s}$ system and $\bar{\Gamma} \equiv\left(\Gamma_{H}+\Gamma_{L}\right) / 2$ denotes their average decay width. The phases $\delta_{1} \equiv \operatorname{Arg}\left(A_{\|}(0)^{*} A_{\perp}(0)\right)$ and $\delta_{2} \equiv \operatorname{Arg}\left(A_{0}(0)^{*} A_{\perp}(0)\right)$ are $C P$-conserving strong phases. In the absence of final-state interactions - probably not a justifiable assumption for $B_{s} \rightarrow J / \psi \phi$ - they are expected to be $0(\bmod \pi)$.

On the other hand, the quantity $\delta \phi=\phi_{\text {CKM }}$ (see Eq. (1)) is a CP-violating weak phase, which is introduced through interference effects between $B_{s}-\overline{B_{s}}$ mixing and decay 


\begin{tabular}{|c|l|}
\hline Observable & Time evolution \\
\hline$\left|A_{0}(t)\right|^{2}$ & $\left|A_{0}(0)\right|^{2}\left[e^{-\Gamma_{L} t}-e^{-\bar{\Gamma} t} \sin (\Delta m t) \delta \phi\right]$ \\
$\left|A_{\|}(t)\right|^{2}$ & $\left|A_{\|}(0)\right|^{2}\left[e^{-\Gamma_{L} t}-e^{-\bar{\Gamma} t} \sin (\Delta m t) \delta \phi\right]$ \\
$\left|A_{\perp}(t)\right|^{2}$ & $\left|A_{\perp}(0)\right|^{2}\left[e^{-\Gamma_{H} t}+e^{-\bar{\Gamma} t} \sin (\Delta m t) \delta \phi\right]$ \\
\hline $\operatorname{Re}\left(A_{0}^{*}(t) A_{\|}(t)\right)$ & $\left|A_{0}(0)\right|\left|A_{\|}(0)\right| \cos \left(\delta_{2}-\delta_{1}\right)\left[e^{-\Gamma_{L} t}-e^{-\bar{\Gamma} t} \sin (\Delta m t) \delta \phi\right]$ \\
$\operatorname{Im}\left(A_{\|}^{*}(t) A_{\perp}(t)\right)$ & $\left|A_{\|}(0)\right|\left|A_{\perp}(0)\right|\left[e^{-\bar{\Gamma} t} \sin \left(\delta_{1}-\Delta m t\right)+\frac{1}{2}\left(e^{-\Gamma_{H} t}-e^{-\Gamma_{L} t}\right) \cos \left(\delta_{1}\right) \delta \phi\right]$ \\
$\operatorname{Im}\left(A_{0}^{*}(t) A_{\perp}(t)\right)$ & $\left.\left|A_{0}(0)\right|\left|A_{\perp}(0)\right| \mid e^{-\bar{\Gamma} t} \sin \left(\delta_{2}-\Delta m t\right)+\frac{1}{2}\left(e^{-\Gamma_{H} t}-e^{-\Gamma_{L} t}\right) \cos \left(\delta_{2}\right) \delta \phi\right]$ \\
\hline
\end{tabular}

Table 3: Time evolution of the decay $B_{s} \rightarrow J / \psi\left(\rightarrow l^{+} l^{-}\right) \phi\left(\rightarrow K^{+} K^{-}\right)$of an initially (i.e. at $t=0$ ) pure $B_{s}$ meson.

\begin{tabular}{|c|c|}
\hline Observable & Time evolution \\
\hline$\left|\bar{A}_{0}(t)\right|^{2}$ & $\left|A_{0}(0)\right|^{2}\left|e^{-\Gamma_{L} t}+e^{-\bar{\Gamma} t} \sin (\Delta m t) \delta \phi\right|$ \\
\hline$\left|\bar{A}_{\|}(t)\right|^{2}$ & $\left.\left|A_{\|}(0)\right|^{2} e^{-\Gamma_{L} t}+e^{-\bar{\Gamma} t} \sin (\Delta m t) \delta \phi\right]$ \\
\hline$\left|\bar{A}_{\perp}(t)\right|^{2}$ & $\left|A_{\perp}(0)\right|^{2}\left[e^{-\Gamma_{H} t}-e^{-\bar{\Gamma} t} \sin (\Delta m t) \delta \phi\right]$ \\
\hline $\operatorname{Re}\left(\bar{A}_{0}^{*}(t) \bar{A}_{\|}(t)\right)$ & $\left|A_{0}(0)\right|\left|A_{\|}(0)\right| \cos \left(\delta_{2}-\delta_{1}\right)\left[e^{-\Gamma_{L} t}+e^{-\bar{\Gamma} t} \sin (\Delta m t) \delta \phi\right]$ \\
\hline $\operatorname{Im}\left(\bar{A}_{\|}^{*}(t) \bar{A}_{\perp}(t)\right)$ & $-\left|A_{\|}(0)\right|\left|A_{\perp}(0)\right|\left[e^{-\bar{\Gamma} t} \sin \left(\delta_{1}-\Delta m t\right)-\frac{1}{2}\left(e^{-\Gamma_{H} t}-e^{-\Gamma_{L} t}\right) \cos \left(\delta_{1}\right) \delta \phi\right]$ \\
\hline $\operatorname{Im}\left(\bar{A}_{0}^{*}(t) \bar{A}_{\perp}(t)\right)$ & $-\left|A_{0}(0)\right|\left|A_{\perp}(0)\right|\left|e^{-\bar{\Gamma} t} \sin \left(\delta_{2}-\Delta m t\right)-\frac{1}{2}\left(e^{-\Gamma_{H} t}-e^{-\Gamma_{L} t}\right) \cos \left(\delta_{2}\right) \delta \phi\right|$ \\
\hline
\end{tabular}

Table 4: Time evolution of the decay $\overline{B_{s}} \rightarrow J / \psi\left(\rightarrow l^{+} l^{-}\right) \phi\left(\rightarrow K^{+} K^{-}\right)$of an initially (i.e. at $t=0$ ) pure $\overline{B_{s}}$ meson.

processes. It can be expressed in terms of elements of the CKM matrix [9, 12] as

$$
\exp (i \delta \phi)=\frac{V_{t s} V_{t b}^{*}}{V_{t s}^{*} V_{t b}} \frac{V_{c s}^{*} V_{c b}}{V_{c s} V_{c b}^{*}}
$$

and is very small, as can be seen easily by applying the Wolfenstein expansion of the CKM matrix [10]. At leading order in this expansion $\delta \phi$ vanishes. However, taking into account higher-order terms (for a treatment of such terms, see e.g. Ref. [42]) gives a non-vanishing result [13, 43]:

$$
\delta \phi=2 \lambda^{2} \eta=\mathcal{O}(0.03) .
$$

Consequently $\delta \phi$ measures simply the CKM parameter $\eta$. Note that $\lambda=\sin \theta_{\mathrm{C}}=0.22$ is related to the Cabibbo angle. Useful expressions for $\delta \phi$ can be found in Ref. [13], where the following relation has been derived:

$$
\delta \phi=2 \lambda^{2} R_{b} \sin \gamma .
$$


Here $\gamma$ is one angle of the "usual" unitarity triangle [44]. Consequently, if the CKMparameter $R_{b}$ (defined by Eq. (6i)) is used as an input, $\delta \phi$ allows a determination of $\gamma$. That input allows, however, also the determination of $\eta$ (or $\gamma$ ) from the mixing-induced $\mathrm{CP}$ asymmetry of $B_{d} \rightarrow J / \psi K_{S}$ measuring $\sin 2 \beta$, where $\beta$ denotes another angle of the unitarity triangle [44]. If one compares these two results for $\eta$ (or $\gamma$ ) obtained from $B_{s}$ and $B_{d}$ modes, respectively, a test of whether the $B_{s}-\overline{B_{s}}$ and $B_{d}-\overline{B_{d}}$ mixing phases are described by the Standard Model, or receive additional contributions from physics beyond that model can be performed. Needless to note, a measurement of a value of $\delta \phi$ much larger than the Standard Model expectation of $\mathcal{O}(0.03)$ would anyway be a striking signal for new physics in $B_{s}-\overline{B_{s}}$ mixing.

An interesting interpretation of $\delta \phi$ has been given in Ref. [45]. There it was shown that $\delta \phi$ is related to one angle in a rather squashed (and therefore "unpopular") unitarity triangle. Note that terms of $\mathcal{O}\left(\delta \phi^{2}\right)$ have been neglected in Table 3 .

The angular distribution for an initially present $\overline{B_{s}}$ meson is given by

$$
\begin{aligned}
& \frac{d^{3} \Gamma\left[\overline{B_{s}}(t) \rightarrow J / \psi\left(\rightarrow l^{+} l^{-}\right) \phi\left(\rightarrow K^{+} K^{-}\right)\right]}{d \cos \theta d \varphi d \cos \psi} \propto \frac{9}{32 \pi}\left[2\left|\bar{A}_{0}(t)\right|^{2} \cos ^{2} \psi\left(1-\sin ^{2} \theta \cos ^{2} \varphi\right)\right. \\
+ & \sin ^{2} \psi\left\{\left|\bar{A}_{\|}(t)\right|^{2}\left(1-\sin ^{2} \theta \sin ^{2} \varphi\right)+\left|\bar{A}_{\perp}(t)\right|^{2} \sin ^{2} \theta-\operatorname{Im}\left(\bar{A}_{\|}^{*}(t) \bar{A}_{\perp}(t)\right) \sin 2 \theta \sin \varphi\right\} \\
+ & \left.\frac{1}{\sqrt{2}} \sin 2 \psi\left\{\operatorname{Re}\left(\bar{A}_{0}^{*}(t) \bar{A}_{\|}(t)\right) \sin ^{2} \theta \sin 2 \varphi+\operatorname{Im}\left(\bar{A}_{0}^{*}(t) \bar{A}_{\perp}(t)\right) \sin 2 \theta \cos \varphi\right\}\right],
\end{aligned}
$$

where the angles are again defined by Eqs. (65) and (66). The time dependence of this rate can be obtained easily with the help of Table 1 , where terms of $\mathcal{O}\left(\delta \phi^{2}\right)$ have been neglected, as in Table 3. In calculating Tables 3 and 1 we have used the fact that $B_{s} \rightarrow J / \psi \phi$ (and $\overline{B_{s}} \rightarrow J / \psi \phi$ ) is dominated to excellent accuracy by a single weak amplitude, as we have seen in Section III. Therefore we have to deal only with mixing-induced $\mathrm{CP}$ violation and there is no direct $\mathrm{CP}$ violation, i.e. $\left|\bar{A}_{0}(0)\right|=\left|A_{0}(0)\right|$, $\left|\bar{A}_{\|}(0)\right|=\left|A_{\|}(0)\right|$ and $\left|\bar{A}_{\perp}(0)\right|=\left|A_{\perp}(0)\right|$.

It is important to note that the mass difference $\Delta m$ can be extracted from timedependent analyses of tagged $B_{s} \rightarrow J / \psi \phi$ data samples [33]. Previous experimental feasibility investigations for the extraction of $\Delta m$ focused entirely on tagged flavourspecific modes of $B_{s}$ mesons [46, 47].

\section{IV-C Untagged decays}

Combining Tables 3 and $\mathbb{t}$, we find that the time evolution of the untagged data sample for $f=J / \psi\left(\rightarrow l^{+} l^{-}\right) \phi\left(\rightarrow K^{+} K^{-}\right)$is given by

$$
\begin{aligned}
& \frac{d^{3} \Gamma[f(t)]}{d \cos \theta d \varphi d \cos \psi} \propto \frac{9}{16 \pi}\left[2\left|A_{0}(0)\right|^{2} e^{-\Gamma_{L} t} \cos ^{2} \psi\left(1-\sin ^{2} \theta \cos ^{2} \varphi\right)\right. \\
& +\sin ^{2} \psi\left\{\left|A_{\|}(0)\right|^{2} e^{-\Gamma_{L} t}\left(1-\sin ^{2} \theta \sin ^{2} \varphi\right)+\left|A_{\perp}(0)\right|^{2} e^{-\Gamma_{H} t} \sin ^{2} \theta\right\}
\end{aligned}
$$




$$
\begin{gathered}
+\frac{1}{\sqrt{2}} \sin 2 \psi\left\{\left|A_{0}(0)\right|\left|A_{\|}(0)\right| \cos \left(\delta_{2}-\delta_{1}\right) e^{-\Gamma_{L} t} \sin ^{2} \theta \sin 2 \varphi\right\} \\
+\left\{\frac{1}{\sqrt{2}}\left|A_{0}(0)\right|\left|A_{\perp}(0)\right| \cos \delta_{2} \sin 2 \psi \sin 2 \theta \cos \varphi\right. \\
\left.\left.-\left|A_{\|}(0)\right|\left|A_{\perp}(0)\right| \cos \delta_{1} \sin ^{2} \psi \sin 2 \theta \sin \varphi\right\} \frac{1}{2}\left(e^{-\Gamma_{H} t}-e^{-\Gamma_{L} t}\right) \delta \phi\right] .
\end{gathered}
$$

Remarkably the time dependence of the untagged rate does not depend on the mass difference $\Delta m$. This feature has been discussed within a more general framework in Ref. [14]. Consequently, whereas $\Gamma_{L}$ and $\Gamma_{H}$ can be determined from the untagged data sample, the extraction of $(\Delta m)_{B_{s}}$ requires tagging. As has already been pointed out in [8], because of the lifetime difference $(\Delta \Gamma)_{B_{s}}$, the untagged decay rate [Eq. (71)] develops an interesting contribution for $t>0$, which is proportional to the CP-violating weak phase $\delta \phi$. It originates from the imaginary parts of the interference terms between $A_{\perp}(t)\left(\bar{A}_{\perp}(t)\right)$ and $A_{\|}^{*}(t)\left(\bar{A}_{\|}^{*}(t)\right), A_{0}^{*}(t)\left(\bar{A}_{0}^{*}(t)\right)$. If $\Delta \Gamma \equiv \Gamma_{H}-\Gamma_{L}$ is in fact sizeable, we are optimistic that it will be possible to measure this effect.

\section{IV-D A closer look at the one-angle distribution}

The full three-angle distributions for tagged and untagged $B_{s} \rightarrow J / \psi\left(\rightarrow l^{+} l^{-}\right) \phi(\rightarrow$ $K^{+} K^{-}$) decays discussed in the previous subsections are quite complicated. A much simpler case arises if we integrate out the two decay angles $\varphi$ and $\psi$ in (64), leading to the following one-angle distribution [33]:

$$
\frac{d \Gamma(t)}{d \cos \theta} \propto\left(\left|A_{0}(t)\right|^{2}+\left|A_{\|}(t)\right|^{2}\right) \frac{3}{8}\left(1+\cos ^{2} \theta\right)+\left|A_{\perp}(t)\right|^{2} \frac{3}{4} \sin ^{2} \theta .
$$

Let us first briefly illustrate the angular moment analysis outlined in Section III for this transparent one-angle distribution. In this case, we have

$$
g^{(1)}(\theta)=\frac{3}{8}\left(1+\cos ^{2} \theta\right), \quad g^{(2)}(\theta)=\frac{3}{4} \sin ^{2} \theta .
$$

Consequently, if we choose

$$
w^{(1)}(\theta)=5 \cos ^{2} \theta-1 \quad \text { and } \quad w^{(2)}(\theta)=2-5 \cos ^{2} \theta,
$$

the orthogonality relation

$$
\int_{-1}^{+1} d(\cos \theta) w^{(i)}(\theta) g^{(k)}(\theta)=\delta_{i k}
$$

is satisfied, and we obtain immediately

$$
\begin{aligned}
\left|A_{0}\left(t_{j}\right)\right|^{2}+\left|A_{\|}\left(t_{j}\right)\right|^{2} & \propto \sum_{i}\left(5 \cos ^{2} \theta_{i}-1\right) \\
\left|A_{\perp}\left(t_{j}\right)\right|^{2} & \propto \sum_{i}\left(2-5 \cos ^{2} \theta_{i}\right),
\end{aligned}
$$


where the summation is over all the events in the same time bin as $t_{j}$.

In the case of the untagged one-angle distribution, the $\Delta m t$ oscillations proportional to the CP-violating weak phase $\delta \phi$ cancel, and the terms (76) and (77) evolve like $\left(\left|A_{0}(0)\right|^{2}+\left|A_{\|}(0)\right|^{2}\right) e^{-\Gamma_{L} t}$ and $\left|A_{\perp}(0)\right|^{2} e^{-\Gamma_{H} t}$, respectively. A fit (now with only one parameter in each time evolution) gives the decay widths $\Gamma_{L}$ and $\Gamma_{H}$ of the CP-even and CP-odd $B_{s}$ mass eigenstates, as well as the CP-even and CP-odd rates $\left|A_{0}(0)\right|^{2}+\left|A_{\|}(0)\right|^{2}$ and $\left|A_{\perp}(0)\right|^{2}$, respectively. For limited statistics, one may want to use time moments 48

$$
f^{(n)}=\int_{0}^{\infty} d t t^{n} f(t)
$$

The weighting-functions method is thus an alternative to the two-bin method suggested in [1, 33. Note that we do not need any a priori information about the relative magnitudes of CP-even and CP-odd amplitudes.

In the case of tagged measurements, the integrated decay rates

$$
\Gamma(t)=\int_{-1}^{+1} d(\cos \theta) \frac{d \Gamma(t)}{d \cos \theta}
$$

evolve in time for intitially present $B_{s}$ and $\overline{B_{s}}$ mesons as

$$
\begin{aligned}
\Gamma(t) \propto & \left(\left|A_{0}(0)\right|^{2}+\left|A_{\|}(0)\right|^{2}\right) e^{-\Gamma_{L} t}+\left|A_{\perp}(0)\right|^{2} e^{-\Gamma_{H} t} \\
& -\left(\left|A_{0}(0)\right|^{2}+\left|A_{\|}(0)\right|^{2}-\left|A_{\perp}(0)\right|^{2}\right) e^{-\bar{\Gamma} t} \sin (\Delta m t) \delta \phi
\end{aligned}
$$

and

$$
\begin{aligned}
\bar{\Gamma}(t) \propto & \left(\left|A_{0}(0)\right|^{2}+\left|A_{\|}(0)\right|^{2}\right) e^{-\Gamma_{L} t}+\left|A_{\perp}(0)\right|^{2} e^{-\Gamma_{H} t} \\
& +\left(\left|A_{0}(0)\right|^{2}+\left|A_{\|}(0)\right|^{2}-\left|A_{\perp}(0)\right|^{2}\right) e^{-\bar{\Gamma} t} \sin (\Delta m t) \delta \phi
\end{aligned}
$$

respectively, where we have used Tables 3 and 4 . Consequently, the time-dependent CP asymmetry arising in the decay $B_{s} \rightarrow J / \psi \phi$ takes the following form:

$$
\begin{aligned}
& a_{\mathrm{CP}}\left(B_{s}(t) \rightarrow J / \psi \phi\right) \equiv \frac{\Gamma(t)-\bar{\Gamma}(t)}{\Gamma(t)+\bar{\Gamma}(t)} \\
& \quad=-\frac{\left|A_{0}(0)\right|^{2}+\left|A_{\|}(0)\right|^{2}-\left|A_{\perp}(0)\right|^{2}}{\left(\left|A_{0}(0)\right|^{2}+\left|A_{\|}(0)\right|^{2}\right) e^{-\Gamma_{L} t}+\left|A_{\perp}(0)\right|^{2} e^{-\Gamma_{H} t}} e^{-\bar{\Gamma} t} \sin (\Delta m t) \delta \phi .
\end{aligned}
$$

Using the quantitative estimates collected in Table 1, we obtain

$$
\frac{\left|A_{\perp}(0)\right|^{2}}{\left|A_{0}(0)\right|^{2}+\left|A_{\|}(0)\right|^{2}}=0.1 \ldots 0.5 \text {. }
$$

Although these estimates suffer from large hadronic uncertainties, they indicate that it may not be justified to neglect the CP-odd contributions proportional to $\left|A_{\perp}(0)\right|^{2}$ in the time-dependent CP asymmetry (82). 


\begin{tabular}{|c|c|}
\hline Observables: $b^{(i)}(t)$ & $w^{(i)}(\theta, \varphi, \psi)$ \\
\hline$\left|A_{0}(t)\right|^{2}$ & $\frac{1}{2}\left[5\left(\cos ^{2} \theta-\sin ^{2} \theta \cos 2 \varphi\right)-1\right]$ \\
$\left|A_{\|}(t)\right|^{2}$ & $\frac{1}{2}\left[5\left(\cos ^{2} \theta+\sin ^{2} \theta \cos 2 \varphi\right)-1\right]$ \\
$\left|A_{\perp}(t)\right|^{2}$ & $2-5 \cos ^{2} \theta$ \\
\hline $\operatorname{Re}\left(A_{0}^{*}(t) A_{\|}(t)\right)$ & $\frac{5}{\sqrt{2}} \sin (2 \psi) \sin (2 \varphi)$ \\
$\operatorname{Im}\left(A_{\|}^{*}(t) A_{\perp}(t)\right)$ & $-\frac{5}{2} \sin (2 \theta) \sin \varphi$ \\
$\operatorname{Im}\left(A_{0}^{*}(t) A_{\perp}(t)\right)$ & $\frac{25}{4 \sqrt{2}} \sin (2 \psi) \sin (2 \theta) \cos \varphi$ \\
\hline
\end{tabular}

Table 5: A set of weighting functions for extracting the observables $b^{(i)}(t)$ of the decays $B_{s} \rightarrow J / \psi\left(\rightarrow l^{+} l^{-}\right) \phi\left(\rightarrow K^{+} K^{-}\right)$and $B \rightarrow J / \psi\left(\rightarrow l^{+} l^{-}\right) K^{*}(\rightarrow \pi K)$.

The coefficient of $\sin (\Delta m t) \delta \phi$ in (82) can be experimentally determined [for instance, from the untagged studies outlined above]. Thus the fundamental weak phase $\delta \phi$ can be cleanly extracted once the $\Delta m t$ oscillations are resolved. Future experiments at the Tevatron and the LHC should be able to achieve this goal. Once the $\Delta m t$ oscillations are traced, one can alternatively perform a tagged, one-angle, time-dependent study to separate the $\mathrm{CP}$-even and $\mathrm{CP}$-odd contributions, from each of which $\delta \phi$ can be directly extracted. The efficient extraction of the various observables depends on the detector configuration, so that other possible variations should be considered. The full angular distributions contain, of course, all the available information, and will be determined eventually.

In order to determine $\delta \phi$ from untagged $B_{s} \rightarrow J / \psi \phi$ decays, where the $\Delta m t$ oscillations cancel, the observables corresponding to the interference terms $\operatorname{Im}\left(A_{\|}^{*}(t) A_{\perp}(t)\right)$ and $\operatorname{Im}\left(A_{0}^{*}(t) A_{\perp}(t)\right)$ must be studied. Valuable information about CP-conserving strong phases can also be obtained, thereby sheding light on the hadronization dynamics of $B_{s} \rightarrow J / \psi \phi$ and the issue of "factorization", which predicts trivial strong phases. A set of weighting functions applicable to this case is given in Table 5 .

\section{IV-E The decay $B \rightarrow J / \psi\left(\rightarrow l^{+} l^{-}\right) K^{*}(\rightarrow \pi K)$}

The angular distribution for $B \rightarrow J / \psi\left(\rightarrow l^{+} l^{-}\right) K^{*}(\rightarrow \pi K)$ takes the same form as Eq. (64) if we use the decay angles specified in Eqs. (65) and (66) with $\phi$ replaced by $K^{*}$ and $K^{+}$replaced by the strange meson.

Using the same angles for $\bar{B} \rightarrow J / \psi\left(\rightarrow l^{+} l^{-}\right) \bar{K}^{*}(\rightarrow \pi \bar{K})$, we obtain the analogous angular distribution to the $\overline{B_{s}} \rightarrow J / \psi\left(\rightarrow l^{+} l^{-}\right) \phi\left(\rightarrow K^{+} K^{-}\right)$case given in Eq. (70). The same weighting functions (see Table 5) can therefore be used to determine the corresponding observables in those decays. The comparison of the observables in these two modes would give us an idea of the extent of $S U(3)$ breaking.

If the $K^{* 0}$ is observed to decay to the CP eigenstate $\pi^{0} K_{S}$, the time evolution of the corresponding three-angle distributions [Eqs. (64) and (70)] is given in Tables 6 and [7, respectively [1]. Tables 6 and 0 assume that the unmixed amplitudes depend on a 


\begin{tabular}{|c|l|}
\hline Observable & Time evolution \\
\hline$\left|A_{0}(t)\right|^{2}$ & $\left|A_{0}(0)\right|^{2} e^{-\Gamma t}[1+\sin (2 \beta) \sin (\Delta m t)]$ \\
$\left|A_{\|}(t)\right|^{2}$ & $\left|A_{\|}(0)\right|^{2} e^{-\Gamma t}[1+\sin (2 \beta) \sin (\Delta m t)]$ \\
$\left|A_{\perp}(t)\right|^{2}$ & $\left|A_{\perp}(0)\right|^{2} e^{-\Gamma t}[1-\sin (2 \beta) \sin (\Delta m t)]$ \\
\hline $\operatorname{Re}\left(A_{0}^{*}(t) A_{\|}(t)\right)$ & $\left|A_{0}(0)\right|\left|A_{\|}(0)\right| \cos \left(\delta_{2}-\delta_{1}\right) e^{-\Gamma t}[1+\sin (2 \beta) \sin (\Delta m t)]$ \\
$\operatorname{Im}\left(A_{\|}^{*}(t) A_{\perp}(t)\right)$ & $\left|A_{\|}(0) \| A_{\perp}(0)\right| e^{-\Gamma t}\left[\sin \left(\delta_{1}\right) \cos (\Delta m t)-\cos (2 \beta) \cos \left(\delta_{1}\right) \sin (\Delta m t)\right]$ \\
$\operatorname{Im}\left(A_{0}^{*}(t) A_{\perp}(t)\right)$ & $\left|A_{0}(0) \| A_{\perp}(0)\right| e^{-\Gamma t}\left[\sin \left(\delta_{2}\right) \cos (\Delta m t)-\cos (2 \beta) \cos \left(\delta_{2}\right) \sin (\Delta m t)\right]$ \\
\hline
\end{tabular}

Table 6: Time evolution of the decay $B_{d} \rightarrow J / \psi\left(\rightarrow l^{+} l^{-}\right) K^{* 0}\left(\rightarrow \pi^{0} K_{S}\right)$ of an initially (i.e. at $t=0$ ) pure $B_{d}$ meson.

\begin{tabular}{|c|l|}
\hline Observable & Time evolution \\
\hline$\left|\bar{A}_{0}(t)\right|^{2}$ & $\left|A_{0}(0)\right|^{2} e^{-\Gamma t}[1-\sin (2 \beta) \sin (\Delta m t)]$ \\
$\left|\bar{A}_{\|}(t)\right|^{2}$ & $\left|A_{\|}(0)\right|^{2} e^{-\Gamma t}[1-\sin (2 \beta) \sin (\Delta m t)]$ \\
$\left|\bar{A}_{\perp}(t)\right|^{2}$ & $\left|A_{\perp}(0)\right|^{2} e^{-\Gamma t}[1+\sin (2 \beta) \sin (\Delta m t)]$ \\
\hline $\operatorname{Re}\left(\bar{A}_{0}^{*}(t) \bar{A}_{\|}(t)\right)$ & $\left|A_{0}(0)\right|\left|A_{\|}(0)\right| \cos \left(\delta_{2}-\delta_{1}\right) e^{-\Gamma t}[1-\sin (2 \beta) \sin (\Delta m t)]$ \\
$\operatorname{Im}\left(\bar{A}_{\|}^{*}(t) \bar{A}_{\perp}(t)\right)$ & $-\left|A_{\|}(0) \| A_{\perp}(0)\right| e^{-\Gamma t}\left[\sin \left(\delta_{1}\right) \cos (\Delta m t)-\cos (2 \beta) \cos \left(\delta_{1}\right) \sin (\Delta m t)\right]$ \\
$\operatorname{Im}\left(\bar{A}_{0}^{*}(t) \bar{A}_{\perp}(t)\right)$ & $-\left|A_{0}(0) \| A_{\perp}(0)\right| e^{-\Gamma t}\left[\sin \left(\delta_{2}\right) \cos (\Delta m t)-\cos (2 \beta) \cos \left(\delta_{2}\right) \sin (\Delta m t)\right]$ \\
\hline
\end{tabular}

Table 7: Time evolution of the decay $\overline{B_{d}} \rightarrow J / \psi\left(\rightarrow l^{+} l^{-}\right) \bar{K}^{* 0}\left(\rightarrow \pi^{0} K_{S}\right)$ of an initially (i.e. at $t=0$ ) pure $\overline{B_{d}}$ meson.

single, unique weak phase, which is justified within the CKM model (see Section II). In these tables, $\Gamma$ and $\Delta m>0$ describe $B_{d} \overline{-B_{d}}$ mixing. They are related to each other through the mixing parameter $x_{d} \equiv(\Delta m / \Gamma)_{B_{d}}$. In analogy to Eq. (67), mixing-induced $\mathrm{CP}$ violation in $B_{d} \rightarrow J / \psi\left(\rightarrow l^{+} l^{-}\right) K^{* 0}\left(\rightarrow \pi^{0} K_{S}\right)$ [1], 49] measures a weak phase $\tilde{\beta}$, which is given by

$$
\exp (-2 i \tilde{\beta})=\frac{V_{t d} V_{t b}^{*}}{V_{t d}^{*} V_{t b}} \frac{V_{c s}^{*} V_{c b}}{V_{c s} V_{c b}^{*}} .
$$

Within the Wolfenstein expansion [10], $\tilde{\beta}$ is equal, to a very good approximation, to the angle $\beta$ of the "standard" (non-squashed) unitarity triangle [44]. Therefore we have not distinguished between $\tilde{\beta}$ and $\beta$ in Tables 6 and 0 .

Whereas the rates for tagged $B_{d} \rightarrow J / \psi K_{S}$ and $\overline{B_{d}} \rightarrow J / \psi K_{S}$ events, which are given by

$$
\begin{aligned}
& \Gamma\left[B_{d}(t) \rightarrow J / \psi K_{S}\right] \propto|A(0)|^{2} e^{-\Gamma t}[1-\sin (2 \beta) \sin (\Delta m t)] \\
& \Gamma\left[\overline{B_{d}}(t) \rightarrow J / \psi K_{S}\right] \propto|A(0)|^{2} e^{-\Gamma t}[1+\sin (2 \beta) \sin (\Delta m t)],
\end{aligned}
$$

allow only the determination of $\sin (2 \beta)$ and of $(\Delta m, \Gamma)_{B_{d}}$, an analysis of the tagged 
three-angle distribution for the decay $B_{d} \rightarrow J / \psi\left(\rightarrow l^{+} l^{-}\right) K^{* 0}\left(\rightarrow \pi^{0} K_{S}\right)$ (and its CPconjugate) yields valuable additional information from the interference terms, as can be seen by looking at Tables 6 and 7 :

- $\operatorname{Re}\left(A_{0}^{*}(t) A_{\|}(t)\right)$ provides additional information on $\cos \left(\delta_{2}-\delta_{1}\right)$.

- $\operatorname{Im}\left(A_{\|}^{*}(t) A_{\perp}(t)\right), \operatorname{Im}\left(A_{0}^{*}(t) A_{\perp}(t)\right)$ provide additional information both on $\sin \delta_{1(2)}$ and $\cos \delta_{1(2)}$ and on $\cos (2 \beta)$. The latter quantity plays an important role to resolve discrete ambiguities in the determination of the CKM angle $\beta$ [50].

Predictions for these observables are given in Table 1 .

The largest data sample for $B_{d} \rightarrow J / \psi K^{* 0}$ is, however, not for $K^{* 0} \rightarrow \pi^{0} K_{S}$, but for $K^{* 0} \rightarrow \pi^{-} K^{+}$. The complete angular distributions and time dependences for the relevant decay modes are given in Appendix A. For charged $B$ decays, the corresponding time and angular distribution is obtained by going to the isospin - related mode and setting $\Delta m=0$. Experimental studies of these decays are very important, since they probe $\sin \left(\delta_{1(2)}\right)$ and non-factorizable terms through the observables corresponding to the left-hand sides of Eqs. (38)-(40) [17, 19, 20]. The relevant information about $\delta_{1}$ and $\delta_{2}$ extracted from these $B$ data samples, "tagged" at the time of decay, can be related to $B_{s} \rightarrow J / \psi \phi$ by using $S U(3)$ flavour symmetry of strong interactions, and allows a determination of $\delta \phi$ from the time evolution of even the untagged rate given by Eq. (71) [8]. This approach does not involve the assumption of factorization, just $S U(3)$ symmetry arguments. Unfortunately the corresponding $S U(3)$-breaking corrections cannot be treated in a quantitative way at present.

\section{The angular distribution of the colour-allowed de- cays $B_{s} \rightarrow D_{s}^{*+} D_{s}^{*-}$ and $B \rightarrow D_{s}^{*+} \bar{D}^{*}$}

The decay of $D_{s}^{* \pm}$ is predominantly electromagnetic, i.e. $D_{s}^{* \pm} \rightarrow D_{s}^{ \pm} \gamma$, whereas $\bar{D}^{*}$ decays also strongly to $\bar{D} \pi$. Therefore the angular distributions of the two decay modes discussed in this section are quite different from each other. The first step in the decay chain is, however, still of the form $P \rightarrow V_{1} V_{2}$ (as in $B_{s} \rightarrow J / \psi \phi$ ) and consequently the terms $A_{0}, A_{\|}, A_{\perp}$ retain the same meanings as in Eq. (36) and the same physical significance as in Sect. IV.

\section{V-A The decay $B_{s} \rightarrow D_{s}^{*+}\left(\rightarrow D_{s}^{+} \gamma\right) D_{s}^{*-}\left(\rightarrow D_{s}^{-} \gamma\right)$}

Applying the same convention as in Ref. [51], we define the coordinate system as follows: in the rest frame of the decaying $B_{s}$ meson, let the directions of motion of $D_{s}^{*+}$ and $D_{s}^{*-}$ be $z^{\prime}$ and $z^{\prime \prime}$, respectively. In the plane transverse to $z^{\prime}$ (or $z^{\prime \prime}$ ), choose any direction as $y^{\prime}$ and $y^{\prime \prime}$. The directions of $x^{\prime}$ and $x^{\prime \prime}$ are then specified uniquely via $x^{\prime}=y^{\prime} \times z^{\prime}$ and $x^{\prime \prime}=y^{\prime \prime} \times z^{\prime \prime}$. Thus, $x^{\prime}$ and $x^{\prime \prime}$ point in opposite directions. Then $\left(\theta^{\prime}, \varphi^{\prime}\right)$ is the direction 


\begin{tabular}{|l|c|}
\hline$b^{(i)}(t)$ & $w^{(i)}\left(\theta^{\prime}, \theta^{\prime \prime}, \chi\right)$ \\
\hline$\left|A_{0}\right|^{2}$ & $(-45 / 92) C\left(\theta^{\prime}, \theta^{\prime \prime}\right)+(245 / 92) \sin ^{2} \theta^{\prime} \sin ^{2} \theta^{\prime \prime}$ \\
$\left|A_{\|}\right|^{2}$ & $(10 / 23) C\left(\theta^{\prime}, \theta^{\prime \prime}\right)-(45 / 46) \sin ^{2} \theta^{\prime} \sin ^{2} \theta^{\prime \prime}+(25 / 4) \sin ^{2} \theta^{\prime} \sin ^{2} \theta^{\prime \prime} \cos 2 \chi$ \\
$\left|A_{\perp}\right|^{2}$ & $(10 / 23) C\left(\theta^{\prime}, \theta^{\prime \prime}\right)-(45 / 46) \sin ^{2} \theta^{\prime} \sin ^{2} \theta^{\prime \prime}-(25 / 4) \sin ^{2} \theta^{\prime} \sin ^{2} \theta^{\prime \prime} \cos 2 \chi$ \\
\hline $\operatorname{Re}\left(A_{0}^{*} A_{\|}\right)$ & $25 \sqrt{2} \sin \theta^{\prime} \sin \theta^{\prime \prime} \cos \theta^{\prime} \cos \theta^{\prime \prime} \cos \chi$ \\
$\operatorname{Im}\left(A_{\|}^{*} A_{\perp}\right)$ & $-(25 / 4) \sin { }^{2} \theta^{\prime} \sin ^{2} \theta^{\prime \prime} \sin 2 \chi$ \\
$\operatorname{Im}\left(A_{0}^{*} A_{\perp}\right)$ & $-25 \sqrt{2} \sin \theta^{\prime} \sin \theta^{\prime \prime} \cos \theta^{\prime} \cos \theta^{\prime \prime} \sin \chi$ \\
\hline
\end{tabular}

Table 8: A set of weighting functions for $B_{s} \rightarrow D_{s}^{*+}\left(\rightarrow D_{s}^{+} \gamma\right) D_{s}^{*-}\left(\rightarrow D_{s}^{-} \gamma\right)$. Here $C\left(\theta^{\prime}, \theta^{\prime \prime}\right)=\left(1+\cos ^{2} \theta^{\prime}\right)\left(1+\cos ^{2} \theta^{\prime \prime}\right)$.

of $D_{s}^{+}$in the rest frame of $D_{s}^{*+}$ in the $\left(x^{\prime}-y^{\prime}-z^{\prime}\right)$ coordinate system, whereas $\left(\theta^{\prime \prime}, \varphi^{\prime \prime}\right)$ is the direction of $D_{s}^{-}$in the rest frame of $D_{s}^{*-}$ in the $\left(x^{\prime \prime}-y^{\prime \prime}-z^{\prime \prime}\right)$ coordinate system.

Since the choice of directions of $y^{\prime}$ and $y^{\prime \prime}$ was completely arbitrary, only the combination $\chi=\varphi^{\prime}+\varphi^{\prime \prime}$ of $\varphi^{\prime}$ and $\varphi^{\prime \prime}$ is physical and these two angles will appear in the angular distribution only through $\chi$. In terms of the momenta of particles, the angles $\theta^{\prime}, \theta^{\prime \prime}$ and $\chi$ can be defined as:

$$
\begin{aligned}
\cos \theta^{\prime}=\mathbf{p}_{D_{s}^{+}}^{\prime} \cdot \mathbf{p}_{D_{s}^{*+}}, \cos \theta^{\prime \prime}=\mathbf{p}_{D_{s}^{-}}^{\prime \prime} \cdot \mathbf{p}_{D_{s}^{*-}} \\
\sin \theta^{\prime} \sin \theta^{\prime \prime} \cos \chi=-\cos \theta^{\prime} \cos \theta^{\prime \prime}-\mathbf{p}_{D_{s}^{+}}^{\prime} \cdot \mathbf{p}^{\prime \prime}{ }_{D_{s}^{-}} \\
\sin \theta^{\prime} \sin \theta^{\prime \prime} \sin \chi=\left(\mathbf{p}_{D_{s}^{+}}^{\prime} \times \mathbf{p}_{D_{s}^{-}}^{\prime \prime}\right) \cdot \mathbf{p}_{D_{s}^{*+}} .
\end{aligned}
$$

The bold-faced quantities are unit three-vectors. The unprimed quantities are measured in the rest frame of $B_{s}$, single-primed quantities in the rest frame of $D_{s}^{*+}$, and doubleprimed quantities in the rest frame of $D_{s}^{*-}$.

In terms of these angles, the angular distribution takes the form

$$
\begin{gathered}
\frac{d^{3} \Gamma}{d \cos \theta^{\prime} d \cos \theta^{\prime \prime} d \chi} \propto \frac{9}{64 \pi}\left\{2\left|A_{0}\right|^{2} \sin ^{2} \theta^{\prime} \sin ^{2} \theta^{\prime \prime}\right. \\
+\frac{1}{2}\left|A_{\|}\right|^{2}\left[\left(1+\cos ^{2} \theta^{\prime}\right)\left(1+\cos ^{2} \theta^{\prime \prime}\right)+\sin ^{2} \theta^{\prime} \sin ^{2} \theta^{\prime \prime} \cos 2 \chi\right] \\
+\frac{1}{2}\left|A_{\perp}\right|^{2}\left[\left(1+\cos ^{2} \theta^{\prime}\right)\left(1+\cos ^{2} \theta^{\prime \prime}\right)-\sin ^{2} \theta^{\prime} \sin ^{2} \theta^{\prime \prime} \cos 2 \chi\right] \\
-\operatorname{Im}\left(A_{\|}^{*} A_{\perp}\right) \sin ^{2} \theta^{\prime} \sin ^{2} \theta^{\prime \prime} \sin 2 \chi+2 \sqrt{2} \operatorname{Re}\left(A_{0}^{*} A_{\|}\right) \sin \theta^{\prime} \sin \theta^{\prime \prime} \cos \theta^{\prime} \cos \theta^{\prime \prime} \cos \chi \\
\left.-2 \sqrt{2} \operatorname{Im}\left(A_{0}^{*} A_{\perp}\right) \sin \theta^{\prime} \sin \theta^{\prime \prime} \cos \theta^{\prime} \cos \theta^{\prime \prime} \sin \chi\right\},
\end{gathered}
$$

where the time dependence of all observables is implicit. It can be read off from Table 3 . The weighting functions are listed in Table 8 .

The angular distribution for the CP-conjugate process $\overline{B_{s}} \rightarrow D_{s}^{*+}\left(\rightarrow D_{s}^{+} \gamma\right) D_{s}^{*-}(\rightarrow$ $\left.D_{s}^{-} \gamma\right)$ is given by 


$$
\begin{gathered}
\frac{d^{3} \Gamma}{d \cos \theta^{\prime} d \cos \theta^{\prime \prime} d \chi} \propto \frac{9}{64 \pi}\left\{2\left|\overline{A_{0}}\right|^{2} \sin ^{2} \theta^{\prime} \sin ^{2} \theta^{\prime \prime}\right. \\
+\frac{1}{2}\left|\overline{A_{\|}}\right|^{2}\left[\left(1+\cos ^{2} \theta^{\prime}\right)\left(1+\cos ^{2} \theta^{\prime \prime}\right)+\sin ^{2} \theta^{\prime} \sin ^{2} \theta^{\prime \prime} \cos 2 \chi\right] \\
+\frac{1}{2}\left|\overline{A_{\perp}}\right|^{2}\left[\left(1+\cos ^{2} \theta^{\prime}\right)\left(1+\cos ^{2} \theta^{\prime \prime}\right)-\sin ^{2} \theta^{\prime} \sin ^{2} \theta^{\prime \prime} \cos 2 \chi\right] \\
-\operatorname{Im}\left(\bar{A}_{\|}^{*} \overline{A_{\perp}}\right) \sin ^{2} \theta^{\prime} \sin ^{2} \theta^{\prime \prime} \sin 2 \chi+2 \sqrt{2} \operatorname{Re}\left({\overline{A_{0}}}^{*} \overline{A_{\|}}\right) \sin \theta^{\prime} \sin \theta^{\prime \prime} \cos \theta^{\prime} \cos \theta^{\prime \prime} \cos \chi \\
\left.-2 \sqrt{2} \operatorname{Im}\left({\overline{A_{0}}}^{*} \overline{A_{\perp}}\right) \sin \theta^{\prime} \sin \theta^{\prime \prime} \cos \theta^{\prime} \cos \theta^{\prime \prime} \sin \chi\right\} .
\end{gathered}
$$

The time evolution of the various quantities is the same as in Table 4 .

As in the case of $B_{s} \rightarrow J / \psi \phi$, the decay $B_{s} \rightarrow D_{s}^{*+} D_{s}^{*-}$ is dominated by a single weak amplitude. Therefore the analysis of the tagged and untagged decays outlined in Sect. IV-B and $\mathbb{E - Q}$ remains valid by replacing $\left(J / \psi, \phi, l^{+}, l^{-}, K^{+}, K^{-}\right) \rightarrow$ $\left(D_{s}^{*+}, D_{s}^{*-}, D_{s}^{+}, \gamma, D_{s}^{-}, \gamma\right)$. Since this process is colour-allowed, factorization is expected to hold more strongly.

\section{V-B The decay $B \rightarrow D_{s}^{*+}\left(\rightarrow D_{s}^{+} \gamma\right) \bar{D}^{*}(\rightarrow \bar{D} \pi)$}

Whereas the decay of the $D_{s}^{*+}$ meson is of the form $V \rightarrow P \gamma$, which has the same angular dependence as $V \rightarrow l^{+} l^{-}$for massless leptons, the $\bar{D}^{*}$ decay belongs to the category $V \rightarrow P_{1} P_{2}$. The net angular distribution should therefore have the same form as that for $P \rightarrow V\left(\rightarrow l^{+} l^{-}\right) V\left(\rightarrow P_{1} P_{2}\right)$. The angular distribution is thus given by Eq. (64), where the definitions of angles are the same as in Eqs. (65) and (66) with $\phi$ replaced by $\bar{D}^{*}$, $l^{+}$replaced by $D_{s}^{+}$, and $K^{+}$replaced by the charmed meson arising from the $\bar{D}^{*}$ decay. The angular distribution for the CP-conjugate decay $\bar{B} \rightarrow D_{s}^{*-}\left(\rightarrow D_{s}^{-} \gamma\right) D^{*}(\rightarrow D \pi)$ is given as in Eq. (70). The weighting functions collected in Table 5 can be used to extract the corresponding observables from experimental data.

At this point, a few comments concerning the time evolution of these angular distributions are in order. Let us first consider decays of neutral $B_{d}$ mesons. Since here the final states are flavour-specific, no interference effects between $B_{d}-\overline{B_{d}}$ mixing and decay processes arise in this case. Consequently, the time evolution of the corresponding observables is only governed by the "mixing" of the initial particle, which is either a pure $B_{d}$ or $\overline{B_{d}}$. For $B_{d} \rightarrow D_{s}^{*+}\left(\rightarrow D_{s}^{+} \gamma\right) D^{*-}(\rightarrow \bar{D} \pi)$ and $\overline{B_{d}} \rightarrow D_{s}^{*-}\left(\rightarrow D_{s}^{-} \gamma\right) D^{*+}(\rightarrow D \pi)$, the time evolution of a generic observable $\mathcal{Q}$ of the angular distribution takes hence the form $\mathcal{Q}(t)=\mathcal{Q}(0) e^{-\Gamma t} \cos ^{2}(\Delta m t / 2)$, while we have $\mathcal{Q}(t)=\mathcal{Q}(0) e^{-\Gamma t} \sin ^{2}(\Delta m t / 2)$ in the case of $B_{d} \rightarrow D_{s}^{*-}\left(\rightarrow D_{s}^{-} \gamma\right) D^{*+}(\rightarrow D \pi)$ and $\overline{B_{d}} \rightarrow D_{s}^{*+}\left(\rightarrow D_{s}^{+} \gamma\right) D^{*-}(\rightarrow \bar{D} \pi)$. The time evolutions of the untagged flavour-specific decays and the related $B^{ \pm}$decays can be obtained straightforwardly from these expressions by setting $\Delta m=0$. 


\section{Summary}

The kinematics of $B$ and $B_{s}$ meson decays into two vector-particles, which both continue to decay through $\mathrm{CP}$-conserving interactions into two lighter particles, involve three independent decay angles. The time evolution of the coefficients of the corresponding angular distributions contains valuable information about the lifetime and mass differences between the $B_{s}$ mass eigenstates $B_{s}^{H}$ and $B_{s}^{L}$, the relative magnitudes and phases of CPodd and CP-even decay amplitudes, and CP-violating effects, including the Wolfenstein parameter $\eta$ and the CKM angle $\beta$. The ratios of these coefficients are estimated by using various form-factor models. Determinations of these time-dependent coefficients will be useful in testing these models and furthermore in determining the extent to which factorization or the $S U(3)$ flavour symmetry of strong interactions hold in these decays.

The observables of the angular distributions can be determined from experimental data by an angular-moment analysis in which the data are weighted by judiciously chosen weighting functions in order to arrive directly at the observables. At times, this permits the extraction of the fundamental CKM parameters. A method applicable to all kinds of angular distributions is indicated, where the weighting functions can be determined without any a priori knowledge of the values of the coefficients. This method is almost as good as the likelihood-fit method for a small number of parameters and is expected to give some reliable results even with low statistics where a likelihood fit to a large number of parameters is inefficient.

The $B_{s}$ meson decays $B_{s} \rightarrow J / \psi \phi, D_{s}^{*+} D_{s}^{*-}$ are considered in the light of a possible width difference $(\Delta \Gamma)_{B_{s}}$. The observables of their angular distributions can be related to those of the decays $B \rightarrow J / \psi K^{*}, D_{s}^{*+} \bar{D}^{*}$ by using the $S U(3)$ flavour symmetry, where $B$ stands for $B_{d}$ or $B^{+}$. The full angular distributions for all these transitions are given explicitly, and the corresponding weighting functions are specified. The time-dependent observables in all these decays provide information about the corresponding values of $\Delta \Gamma$ and $\Delta m$. In addition, the decays of $B_{s}$ mesons inform us about the Wolfenstein parameter $\eta$, while $B_{d} \rightarrow J / \psi K^{*}\left(\rightarrow \pi^{0} K_{S}\right)$ probes the CKM angle $\beta$. Some of the quantities related to the $B_{s}$ case can even be extracted from untagged data samples, where one does not distinguish between initially present $B_{s}$ or $\overline{B_{s}}$ mesons. The comparison between coefficients of angular distributions of $B_{s}$ and $B$ mesons may give us an idea about $S U(3)$-breaking effects, while the comparison of colour-suppressed $(B \rightarrow J / \psi V)$ and colour-allowed $\left(B \rightarrow D_{s}^{* \pm} V\right)$ modes should help in testing the expectation that factorization holds to a greater extent in the latter case.

\section{Acknowledgements}

We are grateful to H.J. Lipkin and J.L. Rosner for previous collaborations. We thank D. Atwood, J. Incandela, R. Kutschke, J. Lewis, M. Neubert, K. Ohl, S. Pappas, M. Schmidt, S. Sen, M. Shochet, W. Wester, H. Yamamoto for useful discussions. This work was supported in part by the Department of Energy, Contract Nos. DE-AC0276CHO3000 and DE FG02 90ER40560. 


\section{Appendix}

\section{A Angular distributions and time dependences for flavour-specific $B_{d} \rightarrow J / \psi\left(\rightarrow l^{+} l^{-}\right) K^{*}\left(\rightarrow K^{ \pm} \pi^{\mp}\right)$ modes}

The angles are defined as in (65), where the $\phi$ meson is replaced by $\stackrel{(-)}{K^{*}}$, and the $K^{+}$ meson by the strange meson in the final state. In order to parametrize the corresponding angular distributions, we use the following combinations of trigonometric functions:

$$
\begin{aligned}
& f_{1}=2 \cos ^{2} \psi\left(1-\sin ^{2} \theta \cos ^{2} \varphi\right) \\
& f_{2}=\sin ^{2} \psi\left(1-\sin ^{2} \theta \sin ^{2} \varphi\right) \\
& f_{3}=\sin ^{2} \psi \sin ^{2} \theta \\
& f_{4}=\sin ^{2} \psi \sin 2 \theta \sin \varphi \\
& f_{5}=(1 / \sqrt{2}) \sin 2 \psi \sin ^{2} \theta \sin 2 \varphi \\
& f_{6}=(1 / \sqrt{2}) \sin 2 \psi \sin 2 \theta \cos \varphi .
\end{aligned}
$$

Taking into account $\left|\overline{A_{f}}\right|=\left|A_{f}\right|$ and using the notation $A_{f} \equiv A_{f}(0)$, where $f \in\{0, \|, \perp\}$, we obtain

$$
\begin{aligned}
& \frac{d^{3} \Gamma\left[B_{d}(t) \rightarrow J / \psi\left(\rightarrow l^{+} l^{-}\right) K^{*}\left(\rightarrow K^{+} \pi^{-}\right)\right]}{d \cos \theta d \varphi d \cos \psi}=\frac{9}{32 \pi} \cos ^{2}\left(\frac{\Delta m t}{2}\right) e^{-\Gamma t} \\
& \times\left\{f_{1}\left|A_{0}\right|^{2}+f_{2}\left|A_{\|}\right|^{2}+f_{3}\left|A_{\perp}\right|^{2}-f_{4} \operatorname{Im}\left(A_{\|}^{*} A_{\perp}\right)+f_{5} \operatorname{Re}\left(A_{0}^{*} A_{\|}\right)+f_{6} \operatorname{Im}\left(A_{0}^{*} A_{\perp}\right)\right\} \\
& \frac{d^{3} \Gamma\left[\bar{B}_{d}(t) \rightarrow J / \psi\left(\rightarrow l^{+} l^{-}\right) \overline{K^{*}}\left(\rightarrow K^{-} \pi^{+}\right)\right]}{d \cos \theta d \varphi d \cos \psi}=\frac{9}{32 \pi} \cos ^{2}\left(\frac{\Delta m t}{2}\right) e^{-\Gamma t} \\
& \quad \times\left\{f_{1}\left|A_{0}\right|^{2}+f_{2}\left|A_{\|}\right|^{2}+f_{3}\left|A_{\perp}\right|^{2}+f_{4} \operatorname{Im}\left(A_{\|}^{*} A_{\perp}\right)+f_{5} \operatorname{Re}\left(A_{0}^{*} A_{\|}\right)-f_{6} \operatorname{Im}\left(A_{0}^{*} A_{\perp}\right)\right\} \\
& \frac{d^{3} \Gamma\left[B_{d}(t) \rightarrow J / \psi\left(\rightarrow l^{+} l^{-}\right) \overline{K^{*}}\left(\rightarrow K^{-} \pi^{+}\right)\right]}{d \cos \theta d \varphi d \cos \psi}=\frac{9}{32 \pi} \sin ^{2}\left(\frac{\Delta m t}{2}\right) e^{-\Gamma t} \\
& \quad \times\left\{f_{1}\left|A_{0}\right|^{2}+f_{2}\left|A_{\|}\right|^{2}+f_{3}\left|A_{\perp}\right|^{2}+f_{4} \operatorname{Im}\left(A_{\|}^{*} A_{\perp}\right)+f_{5} \operatorname{Re}\left(A_{0}^{*} A_{\|}\right)-f_{6} \operatorname{Im}\left(A_{0}^{*} A_{\perp}\right)\right\} \\
& \frac{d^{3} \Gamma\left[\bar{B}_{d}(t) \rightarrow J / \psi\left(\rightarrow l^{+} l^{-}\right) K^{*}\left(\rightarrow K^{+} \pi^{-}\right)\right]}{d \cos \theta d \varphi d \cos \psi}=\frac{9}{32 \pi} \sin ^{2}\left(\frac{\Delta m t}{2}\right) e^{-\Gamma t} \\
& \quad \times\left\{f_{1}\left|A_{0}\right|^{2}+f_{2}\left|A_{\|}\right|^{2}+f_{3}\left|A_{\perp}\right|^{2}-f_{4} \operatorname{Im}\left(A_{\|}^{*} A_{\perp}\right)+f_{5} \operatorname{Re}\left(A_{0}^{*} A_{\|}\right)+f_{6} \operatorname{Im}\left(A_{0}^{*} A_{\perp}\right)\right\}
\end{aligned}
$$




\section{References}

[1] I. Dunietz, H. R. Quinn, A. Snyder, W. Toki and H. J. Lipkin, Phys. Rev. D43, 2193 (1991).

[2] P. Bialas et al., Z. Phys. C57 (1993) 115; J. Hrivnac, R. Lednicky and M. Smizanska, J. Phys. G21 (1995) 629.

[3] Q. Shen et al., Phys. Rev. D52 (1995) 2825.

[4] Review of Particle Physics, Phys. Rev. D54 (1996) 159.

[5] G. Valencia, Phys. Rev. D39 (1989) 3339.

[6] G. Kramer and W.F. Palmer, Phys. Rev. D45 (1992) 193, Phys. Lett. B279 (1992) 181, Phys. Rev. D46 (1992) 2969 and 3197, G. Kramer, W.F. Palmer and H. Simma, Nucl. Phys. B428 (1994) 77; G. Kramer, T. Mannel and W.F. Palmer, Z. Phys.. C55 (1992) 497 .

[7] J.S. Hagelin, Nucl. Phys. B193 (1981) 123; E. Franco, M. Lusignoli and A. Pugliese, ibid. B194 (1982) 403; L.L Chau, W.-Y. Keung and M. D. Tran, Phys. Rev. D27 (1983) 2145; L.L Chau, Phys. Rep. 95 (1983) 1; A.J. Buras, W. Slominski and H. Steger, Nucl. Phys. B245 (1984) 369; M.B. Voloshin, N.G. Uraltsev, V.A. Khoze and M.A. Shifman, Yad. Fiz. 46 (1987) 181 [Sov. J. Nucl. Phys. 46 (1987) 112]; A. Datta, E.A. Paschos and U. Türke, Phys. Lett. B196 (1987) 382; A. Datta, E.A.. Paschos and Y.L. Wu, Nucl. Phys. B311 (1988) 35; M. Lusignoli, Z. Phys. C41 (1989) 645; R. Aleksan, A. Le Yaouanc, L. Oliver, O. Pène and Y.-C. Raynal, Phys. Lett. B316 (1993) 567; I. Bigi, B. Blok, M. Shifman, N. Uraltsev and A. Vainshtein, in B Decays, ed. S. Stone, 2nd edition (World Scientific, Singapore, 1994), p. 132 and references therein; M. Beneke, G. Buchalla and I. Dunietz, Phys. Rev. D54 (1996) 4419.

[8] R. Fleischer and I. Dunietz, Phys. Rev. D55 (1997) 259.

[9] N. Cabibbo, Phys. Rev. Lett. 10 (1963) 531; M. Kobayashi and T. Maskawa, Prog. Theor. Phys. 49 (1973) 652.

[10] L. Wolfenstein, Phys. Rev. Lett. 51 (1983) 1945.

[11] R. Fleischer and I. Dunietz, Phys. Lett. B387 (1996) 361.

[12] D. Du, I. Dunietz, and Dan-di Wu, Phys. Rev. D34 (1986) 3414; I. Dunietz and J.L. Rosner, Phys. Rev. D34 (1986) 1404; Ya.I. Azimov, N.G. Uraltsev and V.A. Khoze, JETP Lett. 43 (1986) 409; I. Dunietz, Ann. Phys. 184 (1988) 350.

[13] I. Dunietz, in Proceedings of the Workshop on B Physics at Hadron Accelerators, Snowmass, Colorado, eds. P. McBride and C. Shekhar Mishra, Fermilab-CONF93/267 (Batavia, 1993), p. 83. 
[14] I. Dunietz, Phys. Rev. D52 (1995) 3048.

[15] D. Fakirov and B. Stech, Nucl. Phys. B133 (1978) 315.

[16] M. Bauer, B. Stech and M. Wirbel, Z. Phys. C29 (1985) 637 and Z. Phys. C34 (1987) 103.

[17] J. Körner and G. Goldstein, Phys. Lett. 89B (1979) 105.

[18] J.D. Bjorken, Nucl. Phys. B (Proc. Suppl.) 11 (1989) 325.

[19] H. Yamamoto, in same Proceedings as in Ref. [13], p. 29.

[20] T.E. Browder, K. Honscheid and D. Pedrini, Annu. Rev. Nucl. Part. Sci. 46 (1996) 395.

[21] R. Aleksan, A. Le Yaouanc, L. Oliver, O. Pène and Y.-C. Raynal, Phys. Lett. B316 (1993) 567.

[22] J. Bijnens and F. Hoogeveen, Phys. Lett. B283, 434 (1992).

[23] J.L. Rosner, Phys. Rev. D42 (1990) 3732.

[24] T. Mannel, W. Roberts, and Z. Ryzak, Phys. Rev. D44 (1991) 18; Phys. Lett. B259 (1991) 359.

[25] H. Georgi, in Proceedings of TASI-91, Boulder, Colorado, 1991, eds. R.K. Ellis, C.T. Hill and J.D. Lykken (World Scientific, Singapore, 1992), p. 589; B. Grinstein, in Proceedings of the Workshop on High Energy Phenomenology, Mexico City, 1991, ed. M.A. Péres and R. Huerta (World Scientific, Singapore, 1992), p. 161; N. Isgur and M.B. Wise, in Heavy Flavours, eds. A.J. Buras and M. Lindner (World Scientific, Singapore, 1992), p. 234; T. Mannel, in Proceedings of the Workshop on QCD, Aachen, Germany, 1992, eds. P.M. Zerwas and H.A. Kastrup (World Scientific, Singapore, 1993), p. 634; M. Neubert, Phys. Rep. 245, 259 (1994); T. Mannel, J. Phys. G21 (1995) 1007; M. Shifman, in Proceedings of TASI-95, Boulder, Colorado, 1995, p. 409, ed. D. E. Soper (World Scientific, Singapore, 1996).

[26] M. Neubert and B. Stech, preprint CERN-TH/97-99, hep-ph/9705292, to appear in Heavy Flavours II, eds. A.J. Buras and M. Lindner (World Scientific, Singapore, 1998).

[27] For a review, see G. Buchalla, A.J. Buras and M.E. Lautenbacher, Rev. Mod. Phys. 68 (1996) 1125.

[28] J. Bartelt et al., Phys. Rev. Lett. 71 (1993) 4111.

[29] A.J. Buras and R. Fleischer, University of Karlsruhe preprint TTP97-15, hepph/9704376, to appear in Heavy Flavours II, eds. A.J. Buras and M. Lindner (World Scientific, Singapore, 1998). 
[30] R. Fleischer, Int. J. Mod. Phys. A12 (1997) 2459.

[31] R. Fleischer, Z. Phys. C58 (1993) 483 and C62 (1994) 81; G. Kramer, W.F. Palmer and H. Simma, Z. Phys. C66 (1995) 429 (see also the article by these authors listed in Ref. [6]).

[32] A.J. Buras, Nucl. Phys. B434 (1995) 606.

[33] A. S. Dighe, I. Dunietz, H. J. Lipkin and J. L. Rosner, Phys. Lett. B369 (1996) 144.

[34] J.M. Soares, Phys. Rev. D53 (1996) 241.

[35] H.-Y. Cheng, Z. Phys. C69 (1996) 647.

[36] CDF Collaboration, F. Abe et al., Phys. Rev. Lett. 75 (1995) 3068.

[37] N. Isgur and M.B. Wise, Phys. Rev. D42 (1990) 2388.

[38] H.-Y. Cheng and B. Tseng, Phys. Rev. D51 (1995) 6259.

[39] J.D. Richman, in Proceedings of the XXVIII International Conference on High Energy Physics - ICHEP96, Warsaw, July 1996, eds. Z. Ajduk and A.K. Wroblewski (World Scientific, Singapore, 1997), p. 143.

[40] M. Neubert, preprint CERN-TH/97-24, hep-ph/9702375, to appear in Heavy Flavours II, eds. A.J. Buras and M. Lindner (World Scientific, Singapore, 1998).

[41] M.E. Luke, Phys. Lett. B252 (1990) 447.

[42] A.J. Buras, M.E. Lautenbacher and G.. Ostermaier, Phys. Rev. D50 (1994) 3433.

[43] I. Dunietz, Ph.D. Thesis, Ann. Phys. 184 (1988) 350, and references therein.

[44] L.L. Chau and W.-Y. Keung, Phys. Rev. Lett. 53 (1984) 1802.; J.D. Bjorken, private communication, 1987; C. Jarlskog and R. Stora, Phys. Lett. 208B (1988) 268.

[45] R. Aleksan, B. Kayser and D. London, Phys. Rev. Lett. 73 (1994) 18.

[46] See the following papers in same Proceedings as in Ref. [13]: D.J. Ritchie et al., p. 357; J.E. Skarha and A.B. Wicklund, p. 361; T.H. Burnett, p. 367; T.J. Lawry et al., p. 371; X. Lou, p. 373; C. Baltay et al., p. 377; K. Johns, p. 383.

[47] See also V. Andreev et al. (The LEP B Oscillations Working Group), "Combined Results on $B^{0}$ Oscillations: Update for the Summer 1997 Conferences", LEPBOSC 97/2, August 18, 1997.

[48] A. S. Dighe and S. Sen, in preparation. 
[49] B. Kayser, M. Kuroda, R.D. Peccei and A.I. Sanda, Phys. Lett. B237 (1990) 508.

[50] A.S. Dighe, I. Dunietz and R. Fleischer, preprint CERN-TH/98-123, FERMILABPUB-98/101-T (1998).

[51] R. Kutschke, An angular distribution cookbook (unpublished). 